\title{
Effect of flood basalt stratigraphy on the phase of seismic waveforms recorded offshore Faroe Islands
}

\author{
Juerg Schuler ${ }^{1}$, Philip A. F. Christie ${ }^{2}$, and Robert S. White ${ }^{1}$
}

\begin{abstract}
The generation of short-period multiples between highly heterogeneous layers of basalt flows can strongly alter transmitted seismic wavefields. These layers filter and modify penetrating waves, producing apparent attenuation and phase changes in the observed waveforms. We investigated the waveform and apparent phase changes of the primary seismic signal using mainly the maximum kurtosis approach. We compared the seismic recordings from two short-offset vertical seismic profiles (VSPs) with synthetic seismograms, generated from sonic logs in the same wells, and we found that short-period multiples cause a rapid broadening of the primary arrivals and strong apparent phase changes within a short depth interval below the top of
\end{abstract}

\begin{abstract}
the basalt flows. Relatively large uncertainties were associated with estimating constant phase shifts of the seismic arrivals within the topmost $250 \mathrm{~m}$ of the basalt sequences, where complex scattering occurred. Within this interval of the Brugdan I well, a phase-only compensation of the first arrivals with a frequency-independent, combined scattering, and intrinsic attenuation operator was unfeasible. At a greater depth, we found that the phase shifts, predicted by a VSP-derived effective $Q$ value, were similar to those estimated from the VSP signals using the kurtosis method. Thus, phase-only compensation with a combined scattering and intrinsic attenuation operator could work well depending on the seismic signal bandwidth and the distribution, depth, and magnitude of the impedance contrasts in the basalt sequence.
\end{abstract}

\section{INTRODUCTION}

In regions where geologic formations are characterized by alternating layers of high- and low-impedance contrasts, seismic penetration of these layers and imaging of the underlying geologic structure can be challenging. Common formations with such properties include sedimentary formations with cyclic, high-contrast bedding, such as coal and evaporite sequences, and basalt flows with interbed sediments. The latter are the subject of this paper.

A wave propagating through such a sequence experiences severe transmission losses at interfaces and multiple scattering between pairs of interfaces. Both alter the first arrival and coda of the wave. Short-period multiples can shape the reflected and transmitted firstarrival wavelet by interference, where layers are thin relative to the dominant wavelength that penetrates them (Widess, 1973). Here, we follow Peacock and Treitel (1969) by defining short-period multiples, as opposed to long-period multiples, as being reverberations that cannot be separated in a correlogram. The term wavelet is used for the compact primary event or signature that is distinguished from the wave coda that includes long-period multiples. The combined effect, where transmission losses and short-period multiples lead to a decrease of primary energy and build-up of coda energy for a wave propagating through thin beds, is known as stratigraphic filtering (Spencer et al., 1977). The qualitative impact of the stratigraphic filter on the transmitted wavelet is a time delay of the peak/ centroid of the arrival and damping of the high frequencies. O'Doherty and Anstey (1971) first study these effects on waves passing through a stack of fine layers. The study was later extended to receivers within the layers and a medium with and without a free surface (Mateeva, 2003). Schoenberger and Levin $(1974,1978)$ and Richards and Menke (1983) perform numerical simulations that showed how transmission loss across a single interface is frequency independent. However, across many thin layers, one can observe only the product of all the (frequency-independent) transmission losses, which has the cumulative effect of removing the high frequencies from the onset of the forward-scattered waveform

\footnotetext{
Manuscript received by the Editor 5 July 2014; revised manuscript received 5 January 2015; published online 20 April 2015

${ }^{1}$ University of Cambridge, Bullard Laboratories, Cambridge, UK. E-mail: schuler@cantab.net; rsw1@cam.ac.uk.

${ }^{2}$ Schlumberger Gould Research, Cambridge, UK. E-mail: pafc1@slb.com.

(C) 2015 Society of Exploration Geophysicists. All rights reserved.
} 
and moving them into the coda. Theoretical approaches were developed later to describe this phenomenon in relation to the statistics of a discontinuous medium (e.g., Banik et al., 1985; Resnick et al., 1986; Burridge et al., 1988; Shapiro and Treitel, 1997).

Burridge (1990) and de Hoop et al. (1991) study the phenomenon of signal broadening with a pulse that traveled through a highly discontinuous medium comprising many homogeneous, horizontal layers. They note that due to the spatial distribution of the reflection and transmission coefficients, the recorded amplitudes of the propagating signal through such a medium is the result of a convolution between the reflection and transmission coefficient series and the source wavelet. Hence, the amplitude at any given frequency depends on the amplitude of the wavelet and the amplitude of the reflection coefficient sequence at the same frequency. The pulse diffused in the experiment about its moving center, whereas the center was delayed relative to the first arrival by the same amount as is predicted by the Backus effective-medium theory (Burridge, 1990). In summary, the studied phenomenon of multiple scattering delays the arrival of the energy peak and leads to apparent attenuation and medium anisotropy (de Hoop et al., 1991).

Schuler et al. (2014) study the apparent attenuation effect of the stratigraphic filter formed by basalt flows. In that paper, a rapid loss of high frequencies with associated pulse broadening was observed with increasing depth below top basalt. We built upon those results by characterizing the phase behavior of the wavelet with depth and by investigating whether we can reproduce the observed features in the vertical seismic profiles (VSPs) by synthetic modeling in an elastic, finely layered medium built from the corresponding welllog data. We show examples from two basalt flow sequences in the Faroe-Shetland Basin that act as stratigraphic filters on the waveforms recorded by two small-offset VSPs.

We have structured the paper such that we show the VSP data first and then match the observations with synthetic seismograms that are based on the borehole logs. We observe rapid source-wavelet broadening below top basalt in the VSP data, and we seek to characterize and reproduce the changes by $1.5 \mathrm{D}$ synthetic modeling. We focus on the apparent phase changes introduced by the basalt sequence. We briefly review what we mean by phase, show a way to estimate it from VSP data, and determine its variation below the top basalt. Synthetic models are used to support the methodology.

\section{DATA ACQUISITION}

High quality data were acquired in two near-vertical wells offshore Faroe Islands on the East Faroe High (Brugdan I well, 6104/21-1; location: $61^{\circ} 16^{\prime} 42.56^{\prime \prime} \mathrm{N}, 4^{\circ} 55^{\prime} 59.36^{\prime \prime} \mathrm{W}$ ) and northwest of the Sjurður High (William well, 6005/13-1A; location: $60^{\circ} 36^{\prime}$ $\left.58.64^{\prime \prime} \mathrm{N}, 5^{\circ} 28^{\prime} 42.89^{\prime \prime} \mathrm{W}\right)$. Both wells were drilled for subbasalt exploration of potential petroleum reservoirs, but economically producible hydrocarbons were not found. At the well sites, the basalt flows can be regarded as near-horizontally layered, although they show rough surfaces on a local scale, sometimes comparable with seismic wavelengths (Jerram et al., 1999; Martini and Bean, 2002).

At Brugdan I, the seabed is at 478-m water depth. The borehole then penetrates $676 \mathrm{~m}$ of Tertiary sediments and $2565 \mathrm{~m}$ of volcanic rocks to reach sediments beneath base basalt. The top of the first basalt flow is at $1176 \mathrm{~m}$ depth below mean sea level. Source signals were generated by a $3 \times 50$-cubic-inch airgun array (140 bar pressure). The array was situated at a $4.5-\mathrm{m}$ water depth and a $75-\mathrm{m}$ offset. The source signals have a peak frequency of approximately $60 \mathrm{~Hz}$ at the hydrophone level (9-m water depth). The source signals were recorded at $1 \mathrm{~ms}$ for a $5 \mathrm{~s}$ record length by downhole geophones with 15.12-m nominal spacing. The three-component (3C) accelerometer geophones have a flat frequency response between 2 and $200 \mathrm{~Hz}$. While drilling and with wireline, petrophysical logs were acquired at $0.1524-\mathrm{m}$ deep intervals. Using the sonic and density logs, the primary reflection coefficient series and power spectrum were calculated and are shown in Figure 1a. The red dots represent the standard deviation of the reflection coefficients within a moving, 50-ms-long time window. Strong reflectivity is observed in the volcanic sequence, where high-impedance basalt cores and hyaloclastites are overlain by low-impedance interflow sediments. In Figure 1b, the power spectrum of the reflection coefficients shows a deficiency at low frequencies, a deficiency also found also in other rock sequences (e.g., Walden and Hosken, 1985). Following Schuler et al. (2014), the Brugdan I volcanic sequence is split into three main depth intervals for our analyses: a first interval (1150-1500 m), comprising high-impedance contrasts that lead to complex wave scattering; a second interval (1500-2300 m); and a third interval (2300-3150 m) that includes fewer high-impedance contrasts per depth unit compared with the first and second interval. We often give more accurate depth specifications in the text because we use selected VSP levels.

The William well is located offshore approximately $110 \mathrm{~km}$ southeast of the Faroe Islands and approximately $80 \mathrm{~km}$ southsouthwest of the Brugdan I well. The sea bottom is at a 742-m water depth, and the borehole penetrates $1100 \mathrm{~m}$ of Tertiary sediments and $1499 \mathrm{~m}$ of a volcanic sequence. The latter comprises mainly basalt flows, mudstones, and hyaloclastites. The borehole is near vertical within the analyzed depth interval. The acquisition design and equipment were the same as for Brugdan I, except that the source offset of the airgun cluster was $50 \mathrm{~m}$. Similarly as at Brugdan I, borehole logs were acquired while drilling and on wire. The reflection coefficient series, its standard deviation as a function of depth, and the associated power spectrum are displayed in Figure 2. Simple basalt flows and interbed mudstones produce a high-impedance sequence between 1950 and $2150 \mathrm{~m}$ depth, whereas a thick tuffaceous and clay-rich mudstone layer leads to a decrease of reflectivity strength between 2150 and $2300 \mathrm{~m}$ depth. Compound basalt flows and mudstone layers are found between 2150 and $2900 \mathrm{~m}$ depth. The final sequence contains mudstones, hyaloclastites, and basalts. Unsurprisingly, the power spectrum of the reflection coefficient series in Figure $2 b$ is deficient at low frequencies.

\section{VERTICAL SEISMIC PROFILE DATA PROCESSING}

The Brugdan I and William VSP survey designs were similar, and thus their data processing steps were basically the same. After removing the gun-tuning shots, the arrival times of the downhole recordings were calibrated for each shot using the hydrophone first breaks. The $3 \mathrm{C}$ recordings of not less than five good-quality shots were then vertically stacked to increase the signal-to-noise ratio $(\mathrm{S} / \mathrm{N})$. An average $\mathrm{S} / \mathrm{N}$ of $139(43 \mathrm{~dB})$ was calculated for the vertical components using the root-mean-square (rms) amplitudes in a 150$\mathrm{ms}$ time window before and after the first breaks. Rotation of the three components to maximum amplitudes along and perpendicular to the ray direction led to little change in the vertical components due to the near-vertical survey design. Traces were then time cor- 
rected for the well deviation $\left(<4^{\circ}\right)$ and source offset using a straightray assumption. Different VSP runs were merged after quality control of the downhole and hydrophone recordings.

Newman's (1973) geometric spreading compensation was then applied to the wavefield before the up and downgoing wavefields were separated by a median filter using 5-9 traces. A low- or bandpass filter usually follows the subtraction process of the median filter to remove high-frequency artifacts that are caused by imperfect source repeatability. We did not need to apply a noise filter, but we did test the impact of different filters on the phase analyses de- a)

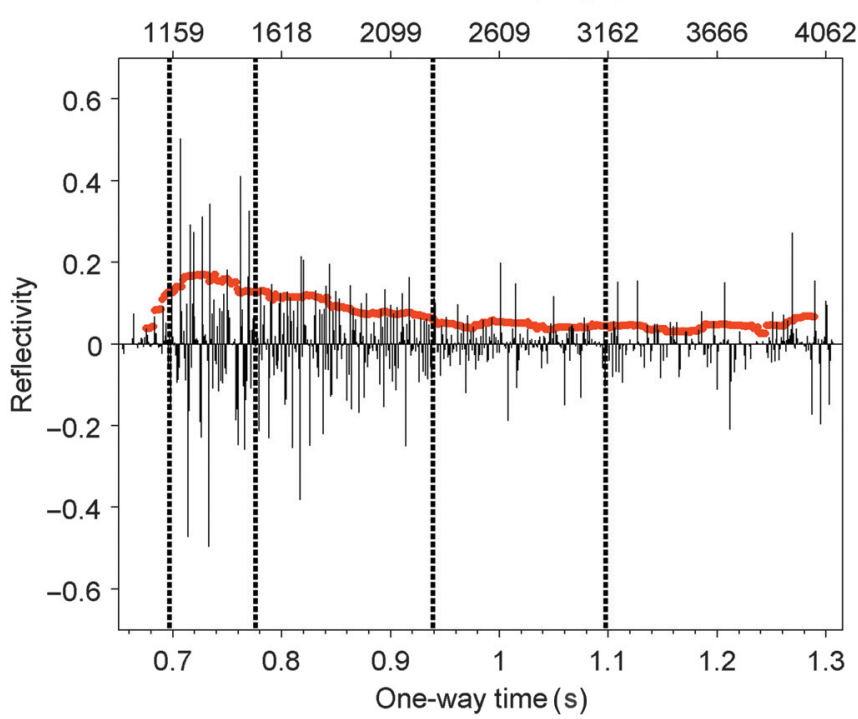

b)

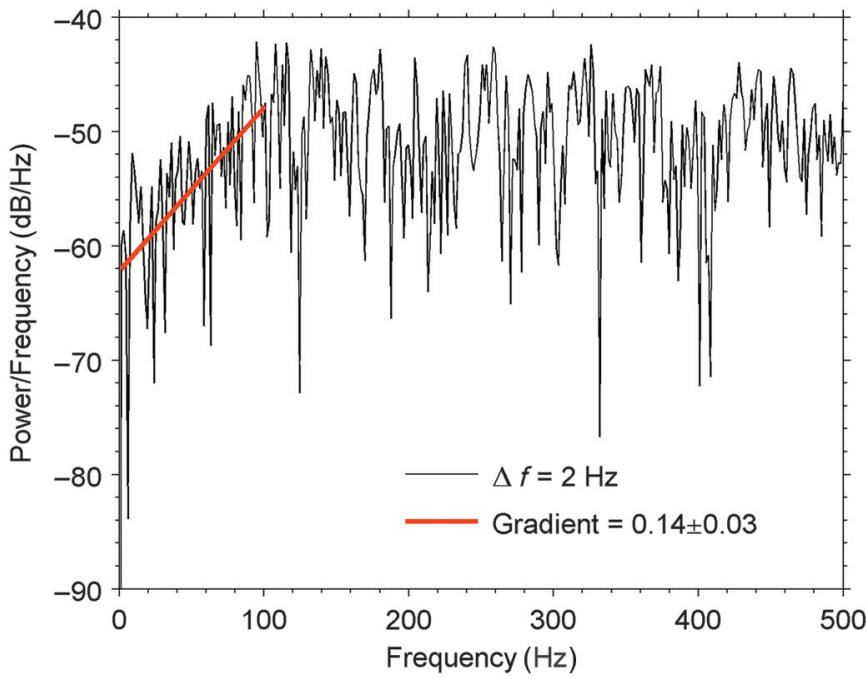

Figure 1. (a) Log-derived reflectivity series of the Brugdan I well versus depth and time. The standard deviations of the reflection coefficients are given as red dots and were calculated for 50-ms-long moving time windows. The vertical dotted lines indicate our analysis depth intervals and (b) the power spectrum of reflectivity series in panel (a). The slope of the reflectivity amplitude spectrum is calculated over the $2-100 \mathrm{~Hz}$ frequency band, and its value is given in the legend with one standard deviation.

a)

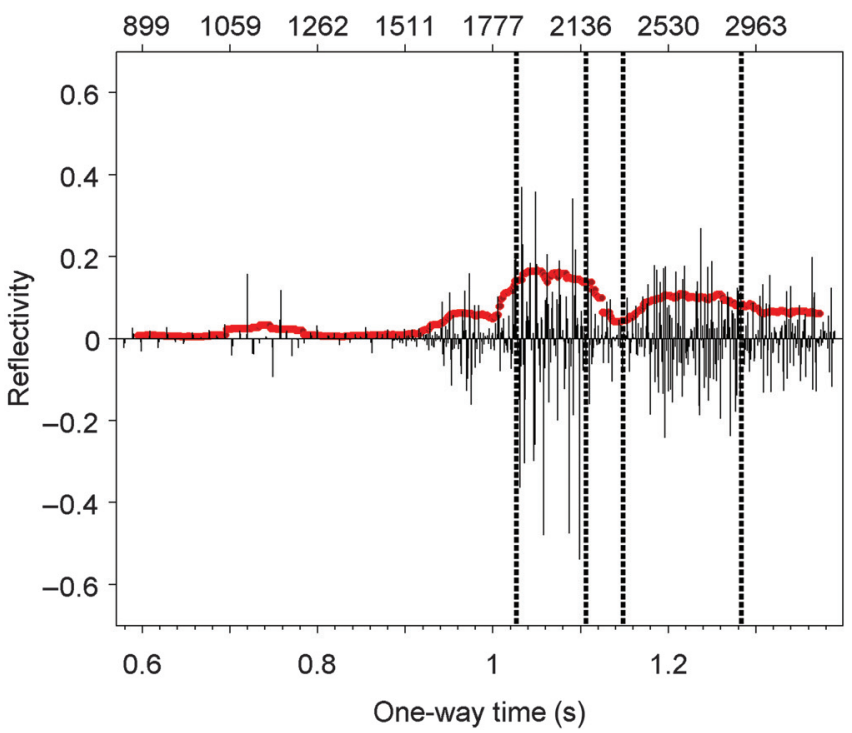

b)

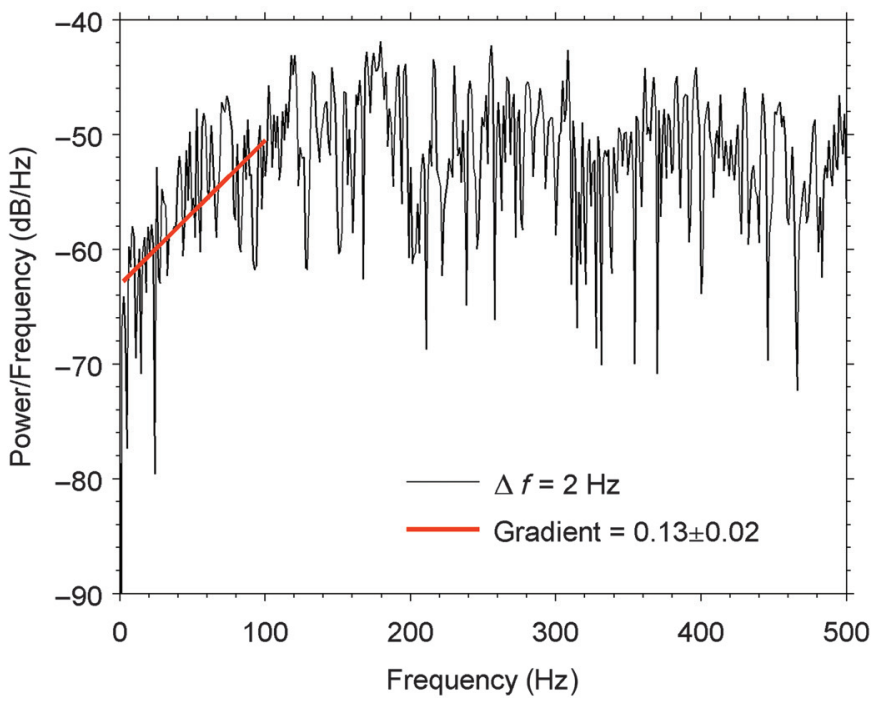

Figure 2. (a) Log-derived reflectivity series of the William well versus depth and time. The standard deviations of the reflection coefficients are given as red dots and were calculated for 50-ms-long moving time windows. Our analysis depth intervals are marked with the vertical dotted lines. (b) The power spectrum of the reflectivity series in panel (a). The slope of the reflectivity amplitude spectrum is calculated over the $2-$ $100 \mathrm{~Hz}$ frequency band, and its value is given in the legend with one standard deviation. 
scribed later. Besides the median filter, we also used a parametric wavefield decomposition technique (Esmersoy, 1990) to verify the impact of the wavefield separation technique on the phase analyses. This inversion technique is amplitude preserving; no prior velocity model and only a few receiver levels are required.

Initially, we have not included a source signature deconvolution of the VSP data because the hydrophone signals are primarily recorded for quality-control purposes, and the signal-to-bubble ratio is already enhanced using an airgun cluster instead of single airguns. Furthermore, the hydrophone signals do not necessarily resemble the far-field signature of the source, and thus they can lead to suboptimal deconvolution results. Nevertheless, we perform a wave-shaping deconvolution on the VSP data as well, using the hydrophone signals as a far-field approximation of the source signals. Ideally, the source deconvolution corrects for pressure variations between shots, increases the efficiency of the vertical stacking, suppresses the bubble effect, and whitens the spectrum. As discussed later, Figure 3 shows the near-field signal recorded with the hydrophone, where the far-field signal was modeled using the Oakwood computing software Gundalf. Also, the downgoing compressional $(\mathrm{P})$ wavefields are shown at 1052 and $1521 \mathrm{~m}$ depths with and without source deconvolution applied.

\section{STRATIGRAPHIC FILTERING EFFECT AT TOP BASALT: OBSERVATIONS}

In the Brugdan I example, the VSP wavefield travels through the sediments above basalt with little change in the waveform, but it is reflected strongly at the topmost basalt flow. Figure 4 displays the normalized traces of the vertical-component seismogram, before and after extracting the downgoing wavefield, together with the velocity and density logs. The trace scaling was evaluated by calculating the rms amplitude of each trace within a 500-ms time window. We observe a rapid broadening of the first-arrival wavelet. High-amplitude peg-leg multiples were generated when the wavefield entered the volcanic sequence. The incident wavefield was multiply scattered at high contrasts of impedance and led to constructive and destructive interference. Short-period multiples are superposed on the primary arrival after its onset to form a broader wavelet after passing through just a few hundred meters of the basalt sequence. We measured a significant drop of the first-arrival amplitude as well as a decrease in the dominant and peak frequencies within this depth interval. Schuler et al. (2014) analyze this in more detail. Although the dominant period of the wavelet above the volcanic rocks is $16 \mathrm{~ms}$, it increases to $42 \mathrm{~ms}$ at $1355 \mathrm{~m}$ depth (approximately $180 \mathrm{~m}$ into the basalt) and changes very little thereafter. A peg-leg multiple generates a dominant double peak in the waveform at $1173 \mathrm{~m}$ depth. It is delayed $11 \mathrm{~ms}$ relative to the first peak and was interpreted as a reflection off the third basalt flow at $1204 \mathrm{~m}$ depth. After Aki and Richards (1980), an average picking error of 1-4 ms was calculated for the vertical components. However, visual guidance of the arrivals for consecutive depth levels leads to smaller
Figure 3. The modeled far-field and recorded (hydrophone) near-field source signals are displayed together with the downgoing P-wavefield at 1052 and $1521 \mathrm{~m}$ depths. The Pwavefields are shown with and without source deconvolution applied to them. All traces were normalized and aligned at $100 \mathrm{~ms}$. The green dot indicates the inflection point tangent pick of the first arrivals.

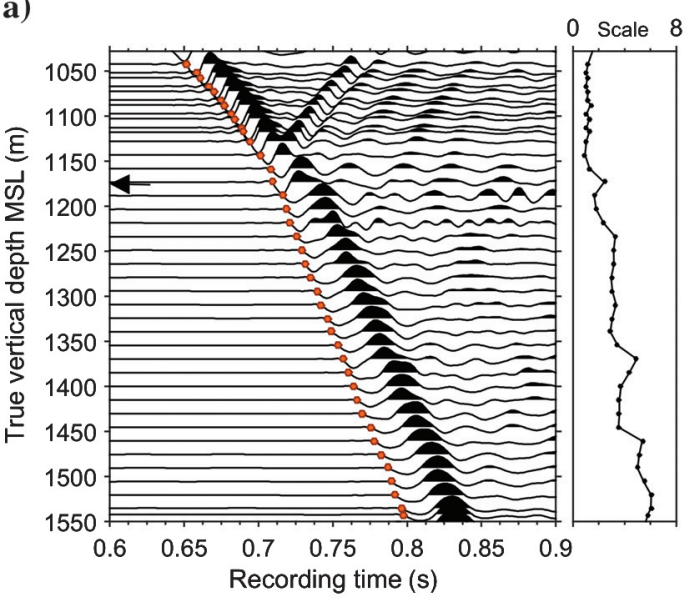

b)

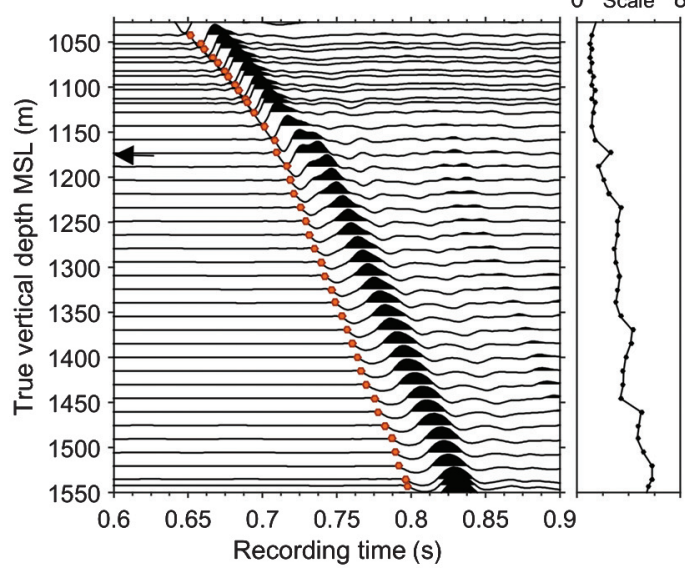

c)

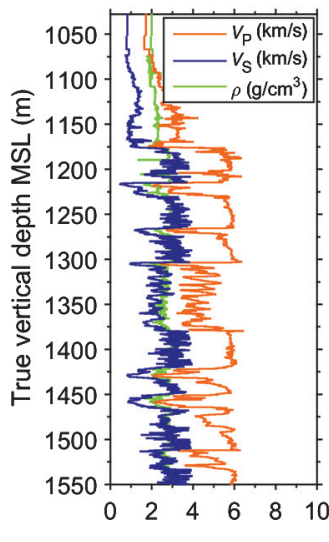

Figure 4. (Brugdan I) Vertical-component VSP seismograms (a) before and (b) after removing the upgoing wavefield from the total wavefield by a seven-trace median filter. The dots represent the picked first-arrival times, and the arrows represent the top basalt depth. For display purposes only, the rms amplitude scaling of the traces is given adjacent to the seismograms and was calculated within a $0.5 \mathrm{~s}$ window. (c) Compressional $\left(V_{\mathrm{P}}\right)$ and shear $\left(V_{\mathrm{S}}\right)$ sonic velocity logs and density $(\rho) \log$. 
picking errors (e.g., Stewart et al., 1984). We estimate our picking accuracy to be $2 \mathrm{~ms}$.

The second VSP example is from the William well, which also shows the effects of signal transmission through basalt flows. Figure 5 shows the normalized traces of the vertical-component seismogram before and after applying a seven-trace median filter that extracted the downgoing wave from the total wavefield. The velocity and density logs are plotted adjacent to the seismograms. Visualizing the downgoing wavefield separately helps to better analyze the waveform changes of the wavelet. It appears as if a second downgoing seismic event merged with the primary peak to form a broader signal after the wave entered the basalts. Within the top $300 \mathrm{~m}$ of the basalt flow succession, the primary wavelet evolved from a wavelet that had its energy focused near the onset to a smooth and more mixed-phase wavelet. A significant change in the dominant period was also measured within this short depth interval. It changed from $18(55 \mathrm{~Hz})$ above the top basalt flow to $40 \mathrm{~ms}(25 \mathrm{~Hz})$ at $2148 \mathrm{~m}$ depth (306 $\mathrm{m}$ into the basalts). The broadening of the first arrival in the William VSP is slower compared with the Brugdan I VSP over the topmost basalt flows. The reflectivity strength (Figure 2) and number of simple basalt flows per unit depth are only slightly higher in the Brugdan I well, but the interbed thicknesses between high-impedance contrasts are often bigger in the William well. The slopes of the reflectivity amplitude spectra are shown in Figures $1 \mathrm{~b}$ and $2 \mathrm{~b}$ over the $2-100 \mathrm{~Hz}$ frequency band. The gradients in Figures $1 b$ and $2 b$ are very similar, but they were computed using the reflectivity series of the entire volcanic sequences. Steeper gradients would suggest that high-impedance contrasts are more frequent across thin layers. A rock sequence with such layering properties leads to more apparent attenuation in the seismic signal. In our case, we expect a steeper gradient in the Brugdan spectrum in the topmost basalt interval. Thus, we calculated the reflectivity amplitude spectra and gradients for the top $400 \mathrm{~m}$ depth interval of the volcanic successions only. We obtained gradients of $0.22 \pm 0.04$ and $0.13 \pm 0.04 \mathrm{~dB} / \mathrm{Hz}$ for the Brugdan I and William spectra over the $2-100 \mathrm{~Hz}$ frequency bands, respectively. The steeper gradient for the Brugdan I spectrum would indeed suggest that more apparent attenuation can be expected, but the error bars of both slopes are relatively large and do not allow us to make further conclusions. Nonetheless, the spatial distribution of the flows and interbed sediments is certainly important in producing different wave interference patterns, which affects the broadening of the first arrival.

Both VSP examples show qualitatively similar waveform modifications when the VSP (airgun) source signal passes through a flood basalt sequence. Attenuation of amplitudes near the onset and the build-up of energy toward the tail of the first arrival further led to a shifting of phase from a front-loaded to a more mixed-phase wavelet. Mechanisms leading to some or all of the observed effects include multiple scattering, transmission losses, and intrinsic attenuation, where seismic wave energy is converted to heat.

\section{STRATIGRAPHIC FILTERING EFFECT AT TOP BASALT: 1D FULL-WAVEFORM MODELING}

We use full-waveform modeling to verify whether the observed waveform changes can be explained by $1 \mathrm{D}$ wave scattering alone. The scattering effects were modeled by a $1.5 \mathrm{D}$ modeling code, which includes 3D wavefield propagation to compute the response of a 1D velocity model. Based on Kennett $(1974,1983)$, the planewave computation of stacked layers is combined with the reflectivity-integral method to give a point-source response. The evolution of the waveform is studied for the Brugdan I and William wells across the top few hundred meters of the basalt sequences using the downgoing VSP signal from above top basalt as the source waveform for modeling. We placed the source at $25 \mathrm{~m}$ offset into a solid medium at $500 \mathrm{~m}$ depth to reduce the number of time samples in the computation. The incidence angle of the wavefront at the top basalt is similar to that in the real VSP.

Borehole logs were scaled to thicker homogeneous layers with the effective-medium technique (Backus, 1962) to understand whether interference alone can cause the observed broadening of the pulse in the VSP seismogram. Synthetic seismograms were computed for models with layer thicknesses of $0.7620,1.5240$, $3.0480,14.9352$, and $24.9936 \mathrm{~m}$, which are all multiples of the 0.1524-m logging interval. The thickness of $0.7620 \mathrm{~m}$ was the a)

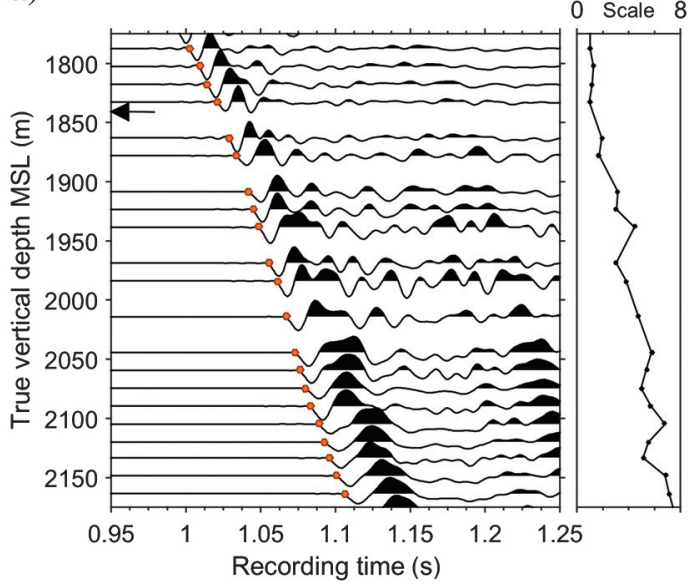

b)

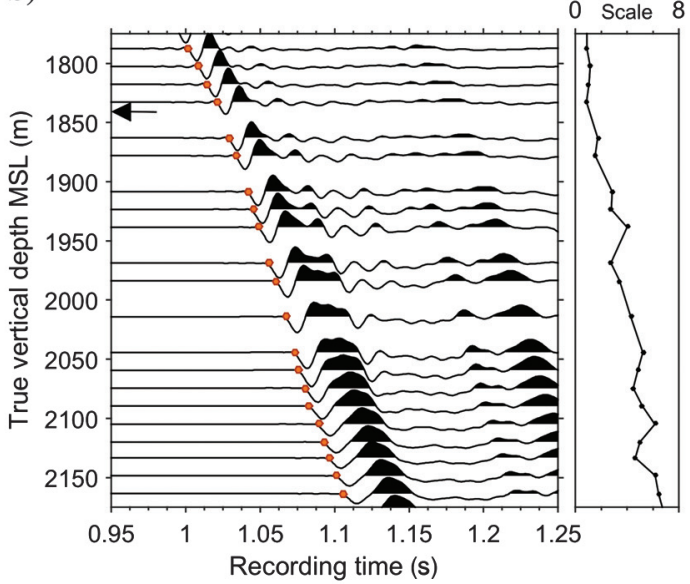

c)

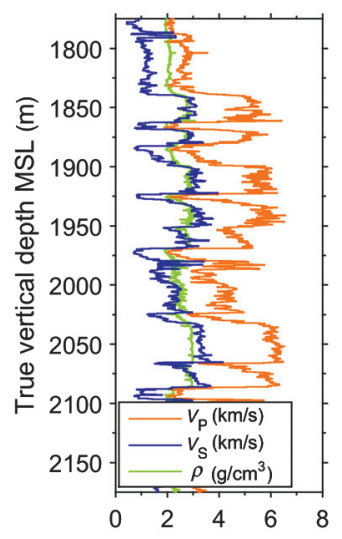

Figure 5. (William) Vertical-component seismograms of the near-offset VSP (a) before and (b) after extracting the downgoing wavefield from the total wavefield by a seven-trace median filter. First breaks are displayed in orange, and the top basalt depth is marked with an arrow. The similar rms amplitude scales of the traces are given adjacent to the seismograms and are computed within a $0.5 \mathrm{~s}$ window. (c) Sonic velocity and density logs. 
smallest layer thickness (model 1) we could run on our computers, and $24.9936 \mathrm{~m}$ (model 2) represents a layer thickness that does not include fine details but still well represents the main velocity variations of the formation. Figure 6 displays the upscaled velocity logs in depth, a proxy for the impedance changes, alongside the resulting synthetic seismograms.

We observe strong peg-leg multiples in model 1 as the wave enters the basalt succession. In fact, a double peak appears already at $1188 \mathrm{~m}$ depth due to the interfering wave that has bounced once within the first basalt flow; the same observation was made in the real data set. The first-arrival amplitudes weaken relative to the peg-leg multiples with increasing depth. Broadening and distortion of the wavelet begin at the top basalt and appear to be completed by $1450 \mathrm{~m}$ depth with little visual change thereafter. The dominant period of the wavelet is $16 \mathrm{~ms}$ above top basalt, $40 \mathrm{~ms}$ at $1355 \mathrm{~m}$ depth, and $42 \mathrm{~ms}$ at $1702 \mathrm{~m}$ depth. The wavelet broadening appears to occur more slowly with depth in the synthetic seismogram than in the real VSP, but the small discrepancy lies within our picking accuracy. We verify that upscaling the model layers slightly, to $0.3048-\mathrm{m}$ thickness instead of $0.7620 \mathrm{~m}$ within a short depth interval across the top basalt, did not yield a more rapid wavelet broadening, although the wave shapes of the synthetic seismograms changed slightly.

Non-1D scattering (e.g., rough surfaces and other lateral heterogeneities), intrinsic attenuation, or both additionally affect the VSP signals. In our examples, we tested only whether their influence is significant. Non-1D scattering and intrinsic loss appear to have a smaller impact on the waveform than 1D scattering because the main waveform features were reproduced in the model 1 seismogram. A waveform feature that could not be reproduced fully within the same depth interval is the relative amplitude of the second trough. We observed a curved reflector in the upgoing VSP wavefield (two-way time) just below top basalt indicating a possible scatterer near the borehole. Such a scatterer could have generated diffractions and refractions that led to wave interference and a more pronounced second trough in our downgoing VSP wavefield. A surface seismic profile shown in Schuler et al. (2014) may support the presence of a potential side structure further because the Brugdan I well was drilled at the edge of a horst-graben structure, where we can expect faults. No other curved reflector was identified down to the base basalt.

In model 2, layers were scaled to 25-m thickness. Superposition of the scattered waves affects the first arrivals but to a lesser degree than in model 1 . The wavelet has not taken on the same smooth and broad shape as rapidly at depth as in model 1 . This is not surprising because less scattering is expected to occur when the layers are thicker and when we upscale the logs. Upscaling has the effect of reducing the impedance contrasts between layers. It also suggests that fine layering indeed is responsible for the broadening of the first arrival. Less scattering was observed with a larger ratio between the wavelength and the characteristic scale length of the medium (layer thickness). Thus, less scattering and little waveform alterations are also observed when the incident wave comprises lower frequencies; i.e., it has a longer wavelength (e.g., Ziolkowski et al., 2003).

To study which interbed layer thicknesses in the volcanic sequence produce wave scattering that leads to a merging double peak in the downgoing first arrival, as observed in the Brugdan I VSP, we arranged two basalt sequences to have 60-, 30-, 15-, 5-, and 1-mthick interbed sediment layers between them. We extracted the characteristic properties of the top two basalt flow sequences from the Brugdan I logs. The extracted compound and simple basalt flows are approximately $40 \mathrm{~m}$ thick each. The background elastic properties were estimated from interbed sediments above, between, and below the extracted basalt sequences. The different model configurations are illustrated in Figure $7 \mathrm{a}$. We then modeled the response of a downgoing source signal traveling through the different models. The source signal above and the response signals below the two basalt sequences are shown in Figure $7 \mathrm{~b}$ for the different model configurations. In all of the resulting seismograms, at least two separate peaks were identified after the first trough, where the scattered waves interact with the primary event. However, the two peaks really start merging when the interbed sediment thickness is less than $5 \mathrm{~m}$, as we see in the Brugdan I downgoing wavefield (Figure 4b). This observation is partly in agreement with Folstad and Schoenberg (1992), who find that scaling layers up to $\lambda / 10$ of the smallest a)

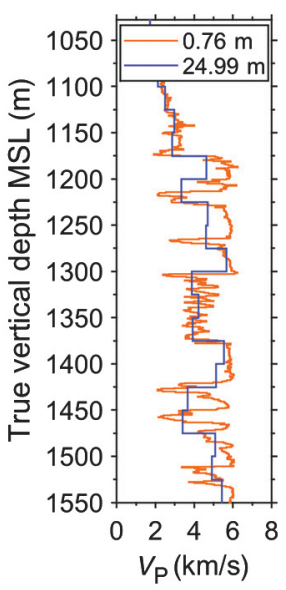

b)

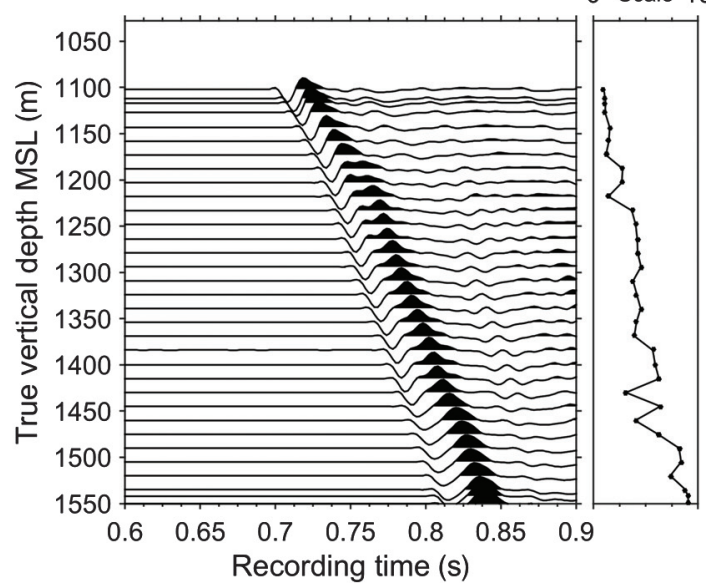

c)

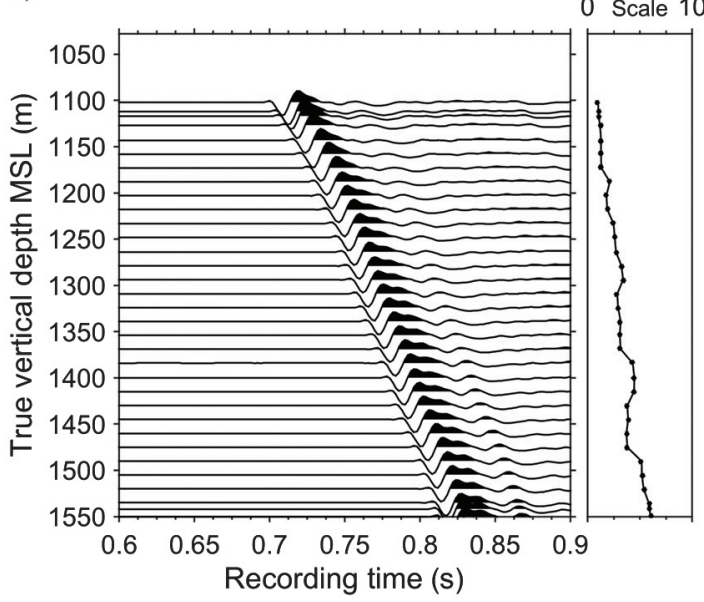

Figure 6. (Brugdan I) Sonic velocity logs (a) scaled from 0.1524 to $0.7620 \mathrm{~m}$ and $24.9936 \mathrm{~m}$. Display of the vertical downgoing wavefield that propagated through the log-derived model scaled to (b) 0.7620 - and (c) $24.9936-\mathrm{m}$-thick layers. The rms amplitude scaling is shown adjacent to the seismic traces and was computed within a $0.5 \mathrm{~s}$ window. 
wavelength yields signals very close to the exact response with insignificant changes in the arrival time, wave shape, or apparent attenuation. Here, the dominant wavelength within the top basalt flow is approximately 100 and $50 \mathrm{~m}$ for interbed sediments. Deeper in the Brugdan I volcanic succession, the dominant wavelengths increase to approximately 200 and $100 \mathrm{~m}$ within the basalt and sediment sections, respectively.

"Layer-cake" models were also built with the William logs and the layers upscaled again to thicknesses of 5, 20, and 98 times the logging interval, to represent the velocity structure of the log. The downgoing and deconvolved VSP waveform from above the top basalt was used as the source wavelet for modeling. Seismogram model 1 in Figure 8b was computed with underlying layers of five times the logging interval. A rapid broadening of the wave occurs across the top basalt. It forms a smooth primary arrival at $2000 \mathrm{~m}$ depth. Its dominant periods at 2148 and $2178 \mathrm{~m}$ depths are 34 $(29 \mathrm{~Hz})$ and $40 \mathrm{~ms}(25 \mathrm{~Hz})$, respectively. The wavelet broadening is delayed relative to the real data. The dominant period of the synthetic first arrival at the $2178 \mathrm{~m}$ depth matches the length of the real wavelet at the $2148 \mathrm{~m}$ depth. In model 2 (Figure 8c), where the transmitted wave propagates through 15-m-thick layers, little change of the arrival shape is observed with depth. a)

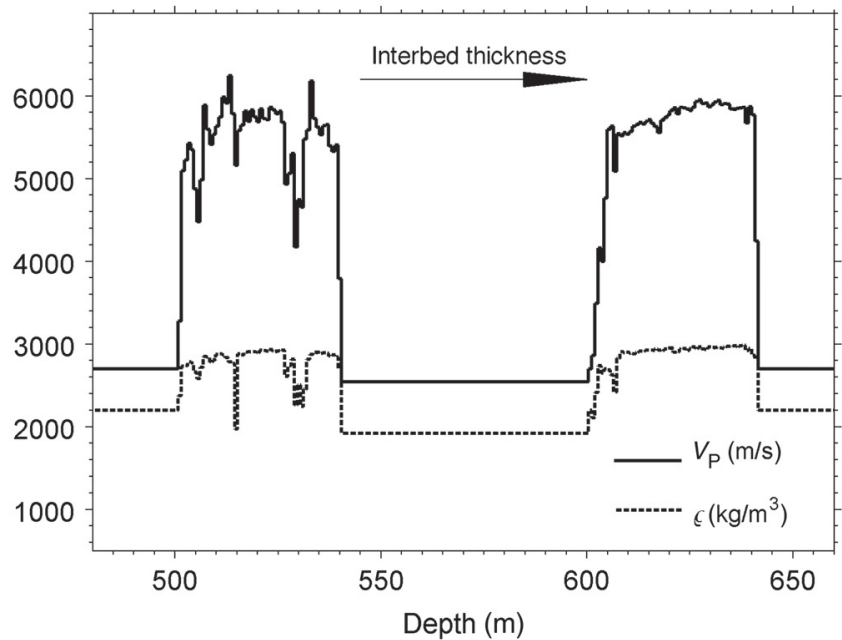

b)

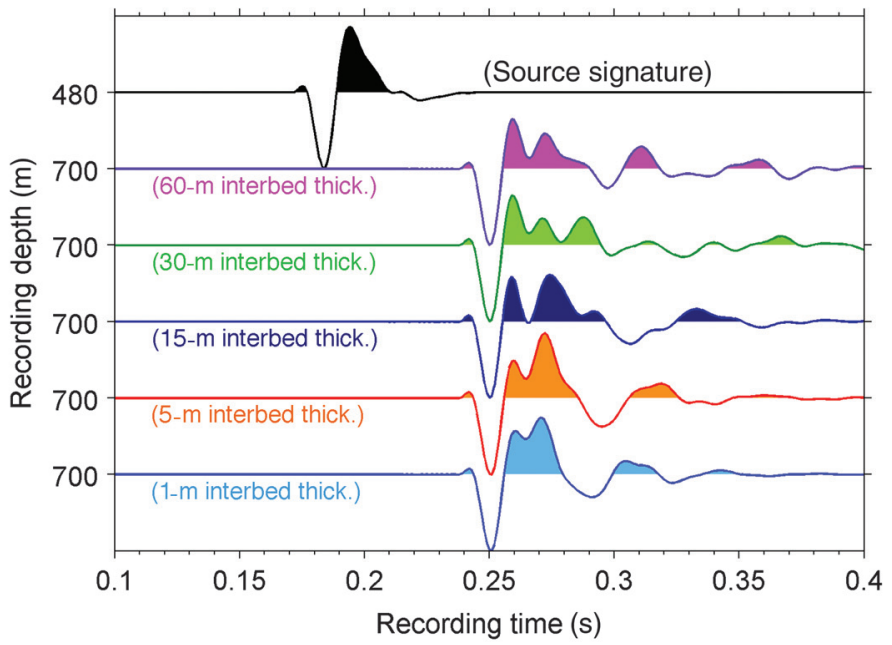

Figure 7. The logs of the two topmost volcanic sequences from the Brugdan I well were extracted. The two sequences are separated by a lowimpedance sedimentary layer. (a) We show P-wave velocity $\left(V_{\mathrm{P}}\right)$ and bulk density $(\rho)$ of the two topmost volcanic sequences. The low-velocity and -density sections above, between, and below the sequences represent characteristic interbed sediment properties. Although the interbed thickness between the two high-impedance sequences is $60 \mathrm{~m}$ in panel (a), we varied the interbed thickness for different modeling runs. The source signal used in the modeling and the downgoing wavefields recorded below the high-impedance sequences is shown in panel (b) for models with different interbed thicknesses.

a)

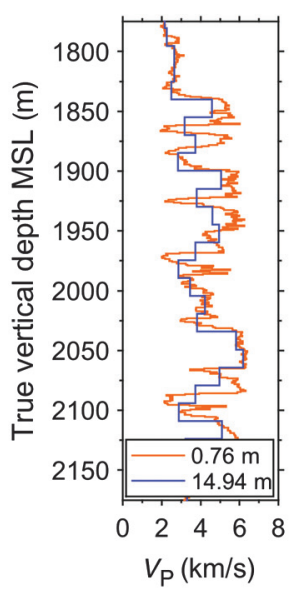

b)

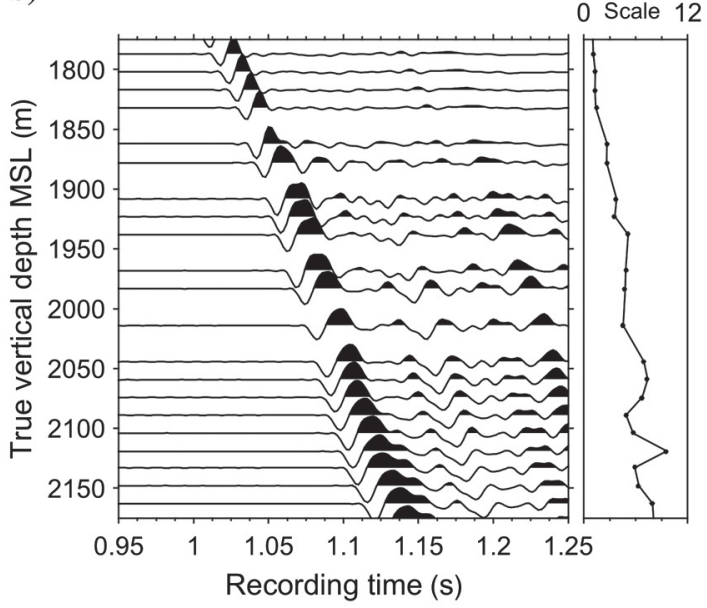

c)

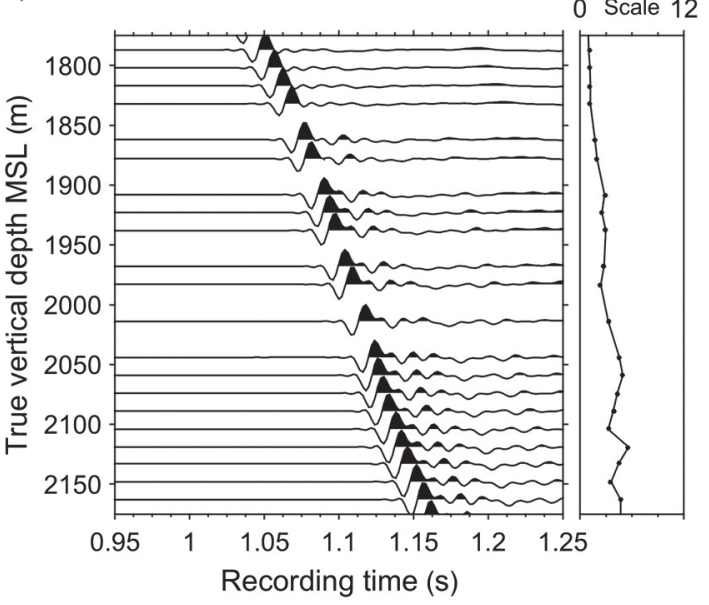

Figure 8. (William) Sonic velocity logs (a) scaled from 0.1524 to $0.7620 \mathrm{~m}$ and $14.9352 \mathrm{~m}$. Display of the vertical downgoing wavefield that propagated through the log-derived model scaled to (b) 0.7620 and (c) $14.9352 \mathrm{~m}$. The rms amplitude scaling is presented adjacent to the seismic traces and was calculated within a $0.5 \mathrm{~s}$ window. 
We conclude from our synthetic simulations that the thin layers with high impedance contrasts have a major impact on the wave shape. In both cases, we could not perfectly match the VSP waveforms with the synthetics, but they showed similar features. Intrinsic attenuation, non-1D scattering, or both are likely to affect the wave propagation as well. Reducing the minimum layer thickness of the model to the logging interval may help in the future to better match the VSP with the synthetic seismogram. For now, we reached a computational limit by including more than 1200 thin layers.

\section{STUDYING THE APPARENT PHASE OF THE DOWNGOING VERTICAL SEISMIC PROFILE WAVEFIELD}

We analyzed the overall shape of the propagating wave through the volcanic sequences in the previous sections. Now, we focus on the apparent phase of the wavelet. The general broadening of the signal signature observed in the VSP wavefield indicates that energy is shifted to later times relative to the first arrival and leads to phase shifts. Therefore, we try to quantify the broadening of the propagating wave by measuring the energy shifts (phase changes) of the first arrivals.

The word "phase" has several meanings depending on context. For a standing oscillating wave, the phase, or more precisely, the "phase offset," is the initial angle where the function starts at the origin. The phase $\phi_{\text {travel }}$ of a traveling wave in time and space is dimensionless and is defined by

$$
\begin{gathered}
u(x, t)=\int_{-\infty}^{\infty} U(0, \omega) e^{i[k(\omega)+i \alpha(\omega)] x+i \phi_{0}} e^{-i \omega t} \mathrm{~d} \omega, \\
u(x, t)=\int_{-\infty}^{\infty} U(0, \omega) e^{-\alpha(\omega) x} e^{i\left[k(\omega) x-\omega t+\phi_{0}\right]} \mathrm{d} \omega, \\
u(x, t)=\int_{-\infty}^{\infty} U(0, \omega) e^{-\alpha(\omega) x} e^{i\left[\phi_{\text {travel }}+\phi_{0]}\right]} \mathrm{d} \omega, \\
u(x, t)=\int_{-\infty}^{\infty} U(x, \omega) \mathrm{d} \omega,
\end{gathered}
$$

where $u$ is the particle displacement of the plane wave, $x$ is the offset, $t$ is the time, $i=\sqrt{-1}, \omega$ is the angular frequency, $\alpha$ is the attenuation factor, $k$ is the wavenumber, $\phi_{\text {travel }}=k(\omega) x-\omega t$ is the phase of the wavelet (Futterman, 1962), and $\phi_{0}$ is the instantaneous or local phase. To understand the local-phase term in equations 1-3, we first consider a standing sine wave, which can be phase shifted to a cosine wave by a $90^{\circ}$ rotation. This can be done by taking the Hilbert transform of a trace $x(t)$ :

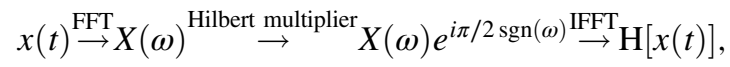

where sgn is the signum function and FFT/IFFT are the forward and inverse fast Fourier transforms, respectively. The rotated function in the time and frequency domains is then given by

$$
\begin{gathered}
x_{\text {rot }}(t)=x(t) \cos (\phi)+\mathrm{H}[x(t)] \sin (\phi), \\
X_{\text {rot }}(\omega)=X(\omega) \cos (\phi)+X(\omega) e^{i \pi / 2 \operatorname{sgn}(\omega)} \sin (\phi), \\
X_{\text {rot }}(\omega)=X(\omega) \cos (\phi)+X(\omega) i \sin (\phi), \\
X_{\text {rot }}(\omega)=X(\omega) e^{i \phi},
\end{gathered}
$$

where $\phi$ is the phase and in equation 8 is given for positive frequencies only. The phase offset is equal to $\phi_{0}$ for a standing wave. In the case of a wavelet at zero time, the phase offset $\phi$ is $\phi_{0}$. Then, $\phi_{0}$ is the instantaneous phase defined, for example, by Bracewell (1965). Even though $\phi$ is dimensionless, it can be expressed as an angle in an Argand (complex plane) diagram. When the discrete signal values of the real and quadrature components are represented in such a plot, the angle of the phasor (rotating vector) to the real axis is the instantaneous or local phase. In geophysics, $\phi_{\text {travel }}+\phi_{0}$ is usually referred to as the phase of a wave. In this section, we investigate $\phi_{0}$ but not $\phi_{\text {travel}}$.

Phase changes (delays or advances) of a wavelet often occur along with rapid changes in the amplitude spectrum and can have various causes (Simm and White, 2002). Levy and Oldenburg (1987) distinguish between phase-changing processes that directly affect the source signature (e.g., dispersion, intrinsic attenuation, processing filters, instrument response, and supercritical reflections) and those that are caused by superposition of scattered waves separated by a time that is short compared with the wavelet duration (i.e., short-period multiples). The latter are referred to as apparent phase changes (Levy and Oldenburg, 1987). In particular, wave interference can cause rapid phase changes of up to $90^{\circ}$ in the presence of thin beds (Edgar and van der Baan, 2011).

Control of the wavelet phase throughout seismic data processing is important because different seismic data sets are frequently compared at the end of the processing chain in a well tie. Several authors (e.g., White, 1988; van der Baan, 2008) have successfully used the maximum kurtosis method to estimate the instantaneous phase and to correct for phase changes in surface seismic data. The underlying assumption is that the phase is independent of the frequency and is stationary within the analysis window. Here, we have adapted this method and applied it to VSP data. A constant $\phi_{0}$ is estimated across the signal bandwidth at different receiver depths and then compared with the phase of the wavelet calculated above the top basalt. We avoid estimating $\phi_{\text {travel }}$ by analyzing the downgoing first arrivals within a time window that is the same relative to the first breaks for all receiver levels. The $\phi_{\text {travel }}$ is therefore taken out by the frame of reference.

\section{KURTOSIS-BASED ESTIMATION OF THE WAVELET PHASE}

We describe next the concept of kurtosis-based phase estimation and mainly follow the work of Levy and Oldenburg (1987), Longbottom et al. (1988), White (1988), Lazear (1993), and van der Baan (2008). Kurtosis is a statistical measure of the peakedness (shape) of a probability distribution of real values (Roenz and Strohe, 1994) and is well suited for estimating constant phase changes of signals within their frequency bandwidths (White, 1988). The assumption of the method is that the reflectivity sequence is white (uncorrelated) and independent (random). Well-log studies have shown that 
the earth reflectivity is generally non-Gaussian and deficient at low frequencies (e.g., Walden and Hosken, 1985, 1986). Thus, primary reflection coefficients are not fully random, although sequences including primaries and internal multiples tend to have a whiter spectrum (White, 1988). The nonwhiteness of the reflectivity series is often considered as a second-order problem (e.g., Ulrych, 1999; van der Baan, 2008).

The main processing steps to obtain a phase estimate are described in the following. Consider a recorded trace $x(t)$, the normalized kurtosis value of a seismic trace with discrete time steps is

$$
K=n \frac{\sum x^{4}(t)}{\left(\sum x^{2}(t)\right)^{2}},
$$

where $K$ stands for the kurtosis value and $n$ is the number of samples. Note that the time series should be as long as possible, so that we can replace the biased sample variance by the population variance. If the kurtosis norm is unity, one single spike is present in the trace; if it is zero, each time sample has a signal of equal amplitude. Donoho (1981) reports that when a white reflectivity series is filtered, the output is nearly Gaussian distributed. Hence, if a white reflectivity series is convolved (i.e., filtered) with an input wavelet, the frequency spectrum becomes more Gaussian (Donoho, 1981). The kurtosis of a Gaussian distributed reflectivity sequence is three. A measure of the deviation of Gaussianity, the excess kurtosis, is obtained by measuring the standardized quantity $D=K-3$. The $D$ is the measure we are after, and we therefore refer to $D$ as the kurtosis (value) from here onward. Additive noise with a Gaussian distribution $(K=3)$ does not affect the phase estimation because it tends toward $D=K-3=0$.

A prerequisite to using kurtosis phase estimation is that the bandwidth of the wavelet exceeds 1.585 octaves, which is equivalent to the statement that the peak frequency must be less than the signal bandwidth (Edgar and van der Baan, 2011). If a spectral notch exists, a $180^{\circ}$ phase jump occurs across the notch (White, 1988). White (1988) notes that even if there is a notch present in the signal bandwidth "this may not greatly upset the standout of the phaseshifted wavelet." We check for $180^{\circ}$ phase jumps at each depth level. Once these conditions are met, the kurtosis is evaluated for predefined phase-angle rotations ranging from $-\pi / 2$ to $\pi / 2$. The trace $x(t)$ is rotated through constant phase angles as shown in equation 7 . The Hilbert operator that is multiplied with the trace is given in the frequency domain by $e^{i \pi / 2 \times \operatorname{sgn}(\omega)}$. In the time domain, it has the form $2 /(\pi t)$. A new signal trace is computed for every rotation parameter $\phi$. The kurtosis value of each new trace is evaluated, and the rotation parameter $\phi$ that leads to a maximum kurtosis value is the most likely phase angle of the signal. The evaluation of the error bar of the phase estimate is not straightforward, but White (1988) gives a comprehensive derivation showing that the error varies inversely to the bandwidth, time duration of the signal to calculate the kurtosis value, the $\mathrm{S} / \mathrm{N}$, and it is also dependent on the spectrum of the subsurface reflectivity.

\section{APPARENT PHASE CHANGES IN THE VERTICAL SEISMIC PROFILE: DATA PREPARATION}

We present the apparent phase-estimation results of the Brugdan I VSP in detail first and then show the results of the William VSP as a second example. The task was to determine the phase shift of the downgoing VSP wave using the maximum kurtosis phase-estimation method. We recognize that basalt sequences are nonstationary, but following van der Baan (2008), we assume that the phase changes are smooth with depth across the seismic bandwidth that we can apply the kurtosis method locally in time windows at each depth level.

We expect that the rapid change in the amplitude spectrum beneath the top basalt introduces a change in the phase spectrum. First, we have to check whether the preconditions hold. Ideally, the time window should be sufficiently long to make the estimation process robust. In other words, a statistical mean can predict more about the data by using a larger amount of data. Downgoing multiples may still be present in the data after applying a median filter. Therefore, we circumvent this issue by choosing window lengths of relatively short time $(100 \mathrm{~ms})$ at the expense of a robust estimation process. Later, we include signal windows of different time lengths to test the robustness of our results. Signals are often filtered during VSP data processing to remove mechanical noise prior to vertical stacking of the seismic traces or to remove subtraction artifacts after the wavefield separation. No noise-cancellation filter had to be applied to the vertical component data. However, we applied zero-phase and minimum-phase filters on the data with different bandwidths (e.g., high-cut $80 \mathrm{~Hz}$, band-pass $4-80 \mathrm{~Hz}$ ) to test their impact on our kurtosis-based phase estimates. No significant changes in the phase estimates were observed. We also compared the phase-estimation results that were obtained from the downgoing wavefields, separated by the median filter, with the phase results of the wavefield separated by the parametric method. No significant phase differences were found for the downgoing wave (vertical geophone component) between the results.

Noise and signal spectra were calculated to check whether the bandwidth condition holds. As an example, the peak frequency of the signal was $46 \mathrm{~Hz}$ just above the top basalt. The low and high cuts of the frequency band were determined by identifying the frequencies at which the peak amplitude dropped by $6 \mathrm{~dB}$ on either side of the peak. Depending on whether or not the notches are included at the low-frequency end of the spectrum, the low-cut frequency is either 7 or $18 \mathrm{~Hz}$. The high-frequency cut is $71 \mathrm{~Hz}$. The effective bandwidth is therefore at least $53 \mathrm{~Hz}$ and fulfills the precondition for robust phase estimation.

White (1988) gives an example for estimating the phase error with a Laplacian distributed reflectivity series that gives a phase error of $11.5^{\circ}$ using 150 time samples and a bandwidth of 0 $125 \mathrm{~Hz}$. Levy and Oldenburg (1987) propose a measure (sensitivity) to evaluate the effects of band-limited data on $D$, where the normalized minimum and maximum kurtosis values give the sensitivity $S_{D}=100\left(D_{\max }-D_{\min }\right) / D_{\max }$. The difference between minimum and maximum kurtosis is a measure of estimation robustness with bigger differences indicating better defined phase estimates (Levy and Oldenburg, 1987). We assessed the variability of our phase-angle estimates by computing the maximum deviation of the phase angles resulting from 100- to 500-ms-long signal windows. The estimates varied more in the topmost basalt interval, where complex scattering is observed.

\section{APPARENT PHASE CHANGES IN THE VERTICAL SEISMIC PROFILE: RESULTS}

Time windows of various lengths were defined to calculate the phase angle for each trace starting at the inflection point tangent 
close to the wavelet onset. We chose a pick location similar to that used by Edgar and Selvage (2011). A minimum time window length of twice the dominant wavelet period of roughly two times $50 \mathrm{~ms}$ at the base basalt was selected for signal analysis. Multiple lengths of this time window were used to calculate phase estimates, with the largest time window being $500 \mathrm{~ms}$ long. It is not necessary to apply a signal taper in time because the complex trace technique analyzes data sample-by-sample. For completeness, it is important to keep in mind that there is always a $180^{\circ}$ ambiguity in each phase-angle estimate and we rearranged them systematically, based on visual inspection of the data with negative phase-angle changes indicating phase delays. When examining the signal signature of the downward-propagating wave at Brugdan I, an apparent phase delay becomes evident between the top basalt, where the energy is initially concentrated at its front end, and the base basalt, where the energy is concentrated more toward the second trough.

Figure 9a shows the apparent phase changes with depth of the downgoing wave at Brugdan I. Beneath the base basalt, wave interference occurs with an oblique arriving wavefront from one side of the well, which leads to an almost maximum-phase first arrival. This is not the interference we like to study, and therefore we disregard any data below $3720 \mathrm{~m}$ depth. The phase changes colored in blue and orange were estimated using the kurtosis and Fourier (details later) analyses, respectively. The sensitivity of the kurtosis value is given at the bottom of Figure 9a. The green squares mark the depth levels in Figure 9a, whose downgoing VSP signals were selected for display in Figure $9 \mathrm{~b}$. We rotated the windowed signals by the estimated phase-shift value relative to the angle of the signal at $1067 \mathrm{~m}$ depth to make a comparison (Figure 9c). The wavelets after rotation in Figure 9c should show a large trough and peak after the first arrivals similar to the reference signal at $1067 \mathrm{~m}$ depth. The procedure of rotating the traces is useful to check whether the phases were estimated well. We disregarded acausal arrivals that

a)

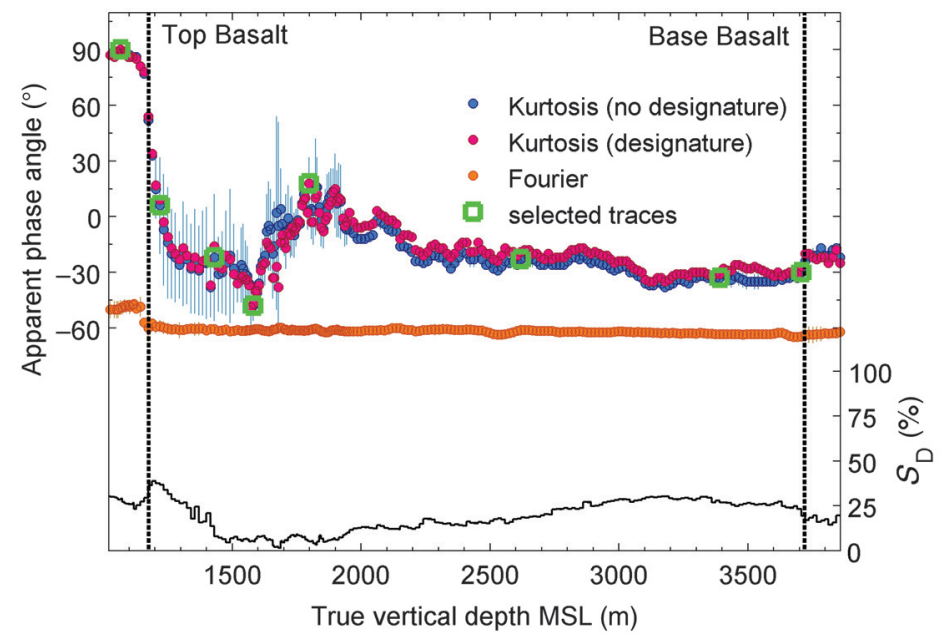

were introduced by the Hilbert transform, $2 /(\pi t)$. Comparing the signals before (Figure 9b) and after phase rotation (Figure 9c), it appears that the kurtosis approach succeeded quite well in bringing the phase back to that of the reference signal disregarding the precursor side lobes. By precursors, we mean the small amplitude peaks before the first troughs in Figure 9c. The precursor side lobes of the rotated traces give the impression that the rotation overcorrected the phase delays. However, we need to keep in mind that strong wave interference occurs near the top of the basalt, which, for example, generated a doublet in the wavelet (Figure 4b). Our kurtosis-model approach is based on a single wavelet propagating through an attenuative/scattering medium, but below the top basalt, we really cannot assume to look at a single first-arriving wavelet anymore. Therefore, we recognize that we ask to estimate the phase of a wavelet that is actually composed of multiple wavelets.

Large phase-estimation uncertainties were calculated in the upper part of the basalt sequence (Figure 9a). Wave interference strongly affects the signal signature and coda in this part and leads to varying phase estimates depending on the time length of the analysis window. Another reason for the high uncertainties in the uppermost part of the basalts may be that the medium is not smooth enough with depth across the sediment-basalt boundary. The reflectivity coefficients are weakly non-Gaussian in this interval, which possibly degrades our kurtosis results and makes the accurate determination of the wavelet phase challenging. Nevertheless, the first arrivals in Figure $9 \mathrm{~b}$ undergo a clear apparent phase change and the kurtosisbased phase estimate appears to be quite reasonable.

The apparent phase changes were also estimated using data in which the source signature was removed prior to vertical stacking of the traces. A very similar phase trend was observed for these data. We have expected similar phase trends between the two differently processed data because short and long time windows during the kurtosis analysis showed little variations in the phase estimates. b)

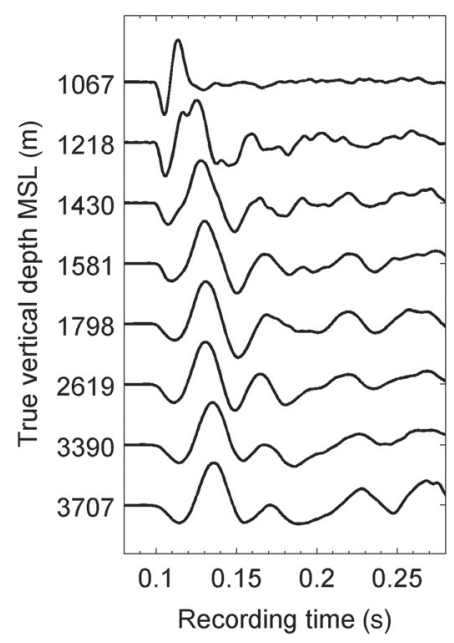

c)

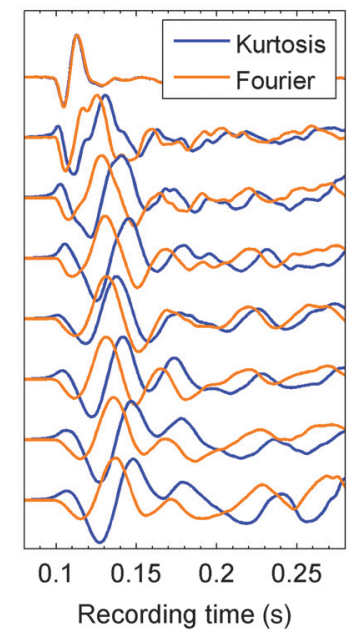

Figure 9. (Brugdan I) (a) The apparent phase estimates were calculated using the kurtosis measure (blue and purple dots) and from Fourier phase spectra (orange dots). In the kurtosis analysis, the phase-angle differences resulting from the longest (500 ms) and the shortest (100 ms) signal windows are displayed with vertical bars at each receiver depth. The label "designature" indicates that the source signature was removed from the data. The sensitivity measure $\left(S_{D}\right)$ of the kurtosis is given at the bottom for the 100-ms signal window. The top and bottom of the volcanic sequence are indicated with a dashed vertical line, and the selected depth levels are shown with green squares. Selected and timealigned downgoing VSP signals are shown in panel (b), and their phase-rotated equivalents are shown in panel (c). The traces were rotated by the estimated phase-shift value in panel (a) relative to the reference level at $1067 \mathrm{~m}$ depth. 
Therefore, the bubble signal did not have a significant impact on the phase estimates.

To illustrate the variation of the kurtosis value $D$ due to different rotation angles of a trace, we prepared the trace, recorded at $2619 \mathrm{~m}$ depth, in Figure 10 and rotated it by selected angles. The traces are labeled with their corresponding rotation angles. The right plot shows the $180^{\circ}$ ambiguity of the maximum and minimum kurtosis value. The peak kurtosis value corresponds to a rotation angle of $-22^{\circ}$, which is used in our phase analysis at $2619 \mathrm{~m}$ depth (Figure 9a).

Constant phase estimates of the time-aligned first arrivals were also calculated from the Fourier phase spectra. The resulting phase angles are plotted in Figure 9a in purple and starting at $-50^{\circ}$ for better visibility. The phases were evaluated at each depth level by finding the peak amplitude spectrum of the primary arrivals and using the unwrapped phase value at that frequency. Depending on the complexity of the phase spectrum, this is usually a good firstorder approach to estimate the average phase of a wavelet. Although the shape of the phase angles was similar to the kurtosis-based curve in Figure 9a, the phase changes are much smaller. A maximum change of only $15^{\circ}$ between top and base basalt was computed using the Fourier spectra. Estimation of phase changes using relatively short signals, however, is challenging because of their low spectral resolution. On the other hand, estimating phase from long time sequences gives a higher spectral resolution, but spectral notches may then degrade the results. In Figure 9c, we used the phase shifts, calculated with the Fourier approach, and rotated the signals back to the reference level. The result shows that the rotation did not bring the energy back to the front end of the signal.

The same phase analysis was performed for the William VSP using identical parameterizations. The data met the preconditions required for a robust estimation. The results of the phase analysis and a few selected traces are displayed in Figure 11. Again, short-period multiples cause wave interference effects when entering the layered basalt sequence. The top basalt is marked with a vertical dashed line in Figure 11a. The phase-angle estimates were rearranged after visual inspection of the signals due to the $180^{\circ}$ ambiguity. We find it more difficult to arrange the kurtosis-based phase angles systematically in this data set compared with the Brugdan I data. The abrupt phase jump at $2120 \mathrm{~m}$ depth emphasizes this. The phase angles could also be subtracted by $180^{\circ}$ below $2120 \mathrm{~m}$ depth. However, a $150^{\circ}$ phase change would lead to a polarity flip of the first arrivals. Another phase jump is observed at $2751 \mathrm{~m}$ depth. The borehole was side tracked between 2558 and $3351 \mathrm{~m}$ depth, and it exhibits a

Figure 10. (a) The vertical signal from the Brugdan I VSP at $2619 \mathrm{~m}$ depth was rotated for selected angles and (b) the kurtosis values of the rotated traces in panel (a) and all other rotation angles between $-180^{\circ}$ and $180^{\circ}$ are shown. The maximum kurtosis value (rotation angle $-22^{\circ}$ ) represents the most likely phase estimate of that trace. It also shows that there is a $180^{\circ}$ ambiguity in determining the rotation angle.

a)

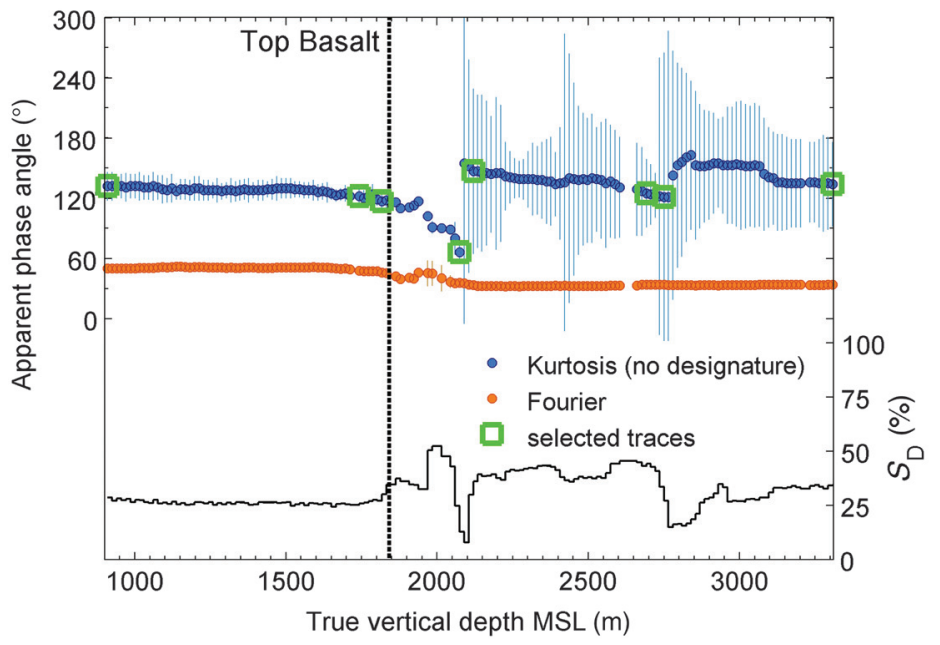

b)

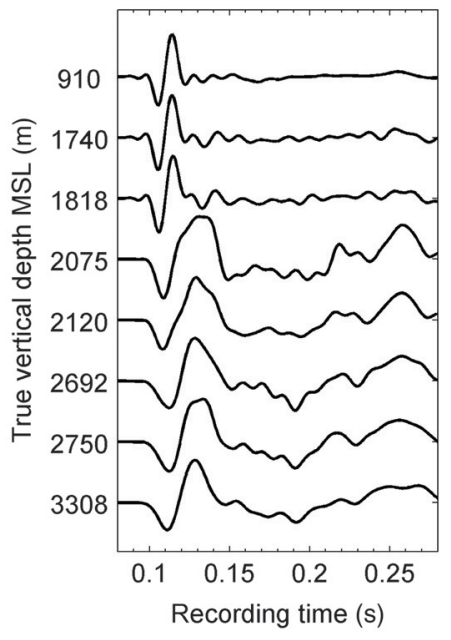

c)

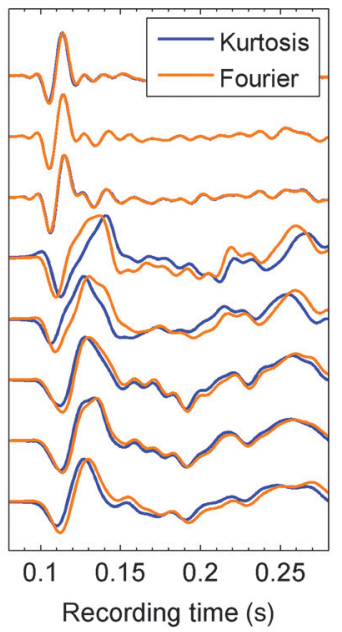

Figure 11. (William) (a) Apparent phase angles were estimated by the kurtosis measure (blue) and Fourier phase spectra (orange). The variance of the phase-angle estimates obtained with a 500- and 100-ms time window starting from the first breaks are displayed with vertical bars. The top of the volcanic sequence is indicated with a dashed vertical line, and selected depth levels are indicated with green squares. The signals of the selected depth levels were time aligned and plotted in panel (b). The kurtosis sensitivity measure $S_{D}$ is given at the bottom for the 100 -ms signal window. The signals in panel (b) were phase rotated by the amount estimated in panel (a) and displayed in panel (c). 
maximum well deviation of $15.3^{\circ}$ between 2607 and $2906 \mathrm{~m}$ depth. We rotate the $3 \mathrm{C}$ geophones to account for the well deviation, but the wavefield still shows unexplained features, which possibly affects our phase measurements. Problems in calculating a seismic quality factor within this interval were also reported by Schuler et al. (2014). Therefore, we disregard the 2607-2906 m depth interval. The phase curve based on the Fourier spectra shows a $20^{\circ}$ drop in the topmost $250 \mathrm{~m}$ of the high-impedance contrast sequence with little change before or after this interval. None of the signals rotated by the evaluated phase shifts in Figure 11a lead to a significantly different first-arrival shapes (see Figure 11b). The different apparent phase curves of the William data cannot be solely explained by the slightly lower reflectivity strength and number of basalt flows per unit depth relative to Brugdan I. Thus, the spatial distribution of the impedance contrasts plays an important role to what extent the wavefield (phase) is altered with depth.

\section{PHASE ESTIMATES OF SYNTHETIC FIRST ARRIVALS: PREPARATIONS}

In this section, we calculated phase estimates from synthetic seismograms based on the Brugdan I well logs. We repeated the previously described 1D full-waveform modeling with different source wavelets to study the apparent phase changes of wavelets with different signal bandwidths. Ricker wavelets, Ormsby wavelets, and two extracted VSP signals from the downgoing wavefield above the top basalt were prepared as source waveforms for the modeling. The acausal and zero-phase wavelets were time shifted to be causal and shaped to front-loaded signals with the Kolmogorov method (Claerbout, 1985). Both synthetic wavelet types are convenient for studying phase changes due to their characteristic frequency spectra. For example, the signal with corner frequencies of $1-$ $15-30-50 \mathrm{~Hz}$ has a bandwidth close to an airgun signature that is used to image subbasalt structures. The wavelets were shaped to front-loaded signals to resemble physical source signals as produced, for example, by airguns. The first breaks of the front-loaded wavelets and extracted VSP signals were picked at the inflectionpoint tangents.

\section{PHASE ESTIMATES OF SYNTHETIC FIRST ARRIVALS: RESULTS}

Figure 12a illustrates the apparent phase estimates that were obtained by propagating different source waveforms through the Brugdan I model. For the sake of visualization, the apparent phases of the input wavelets were aligned at $90^{\circ}$. Figure $12 \mathrm{~b}$ shows the input wavelets at $1000 \mathrm{~m}$ depth, and Figure 12c displays the first arrivals at $1899 \mathrm{~m}$ depths for comparison. They were all amplitude normalized and time aligned at the vertical dashed line. Three frontloaded wavelets with the same $1 \mathrm{~Hz}$ low-cut but different high-cut frequencies were also prepared. The more high-frequency content the wavelets have, the stronger the apparent phase changes, especially within the top few hundred meters of the basalts. These apparent phase changes with depth appear to be frequency dependent; i.e., we observe dispersion. Burridge et al. (1988) show that thinbed scattering can lead to apparent dispersion with an effective wavelet that broadened about the pulse centroid, according to a diffusion equation, while propagating through many thin layers. Based on Figure 12a, their apparent phases appear to change little below approximately $1300 \mathrm{~m}$ depth. The extracted VSP signal and two a)

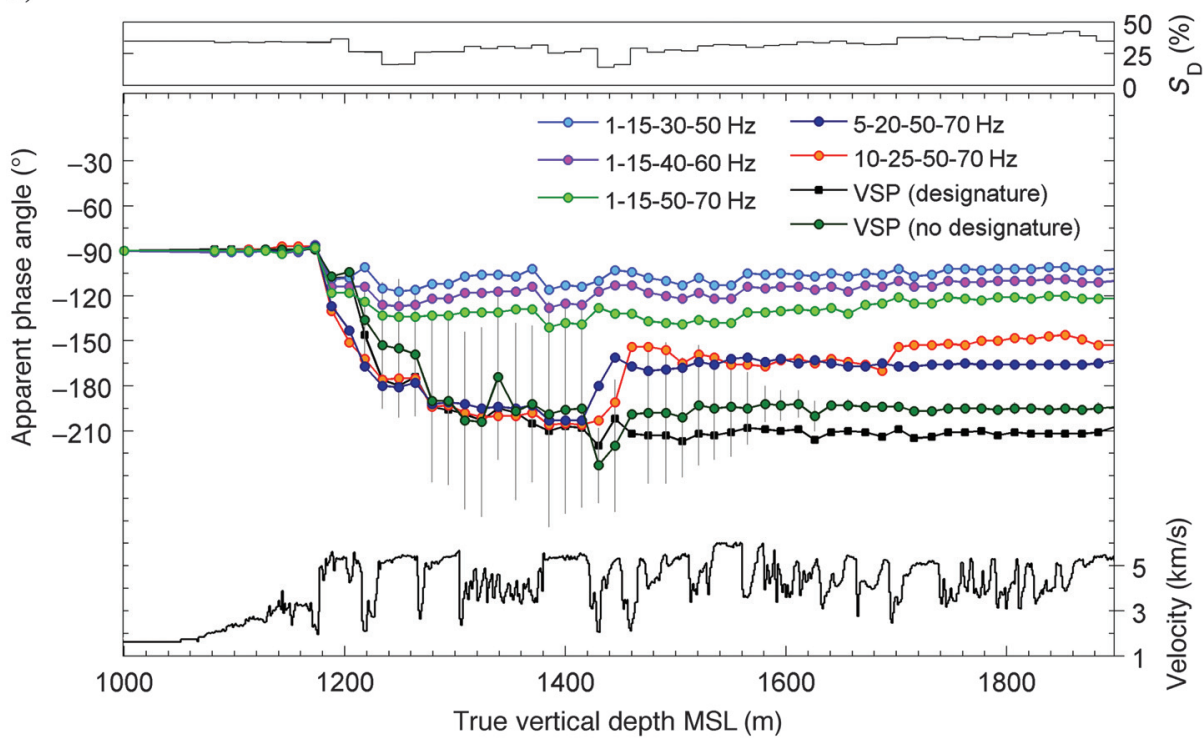

b)

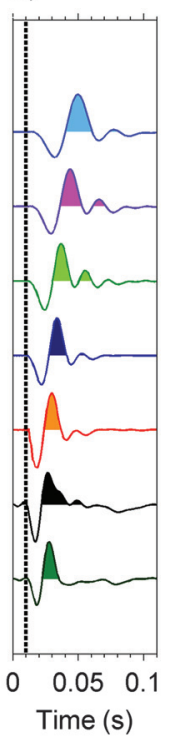

c)

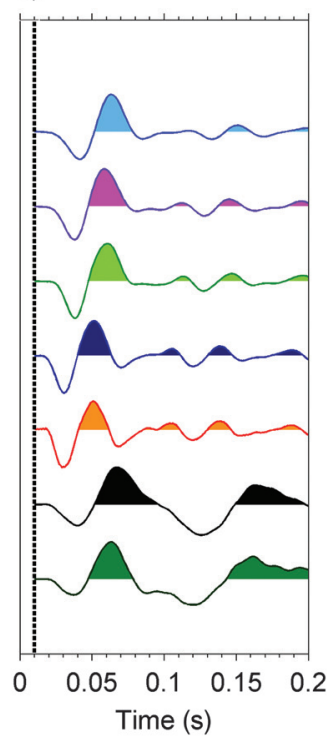

Figure 12. (a) Phase-angle estimates of the synthetic seismograms are shown, in which we let a wavelet propagate through the basalt sequence. The four corner frequencies of the Ormsby wavelets at $1000 \mathrm{~m}$ depth are specified in the legends before they were shaped to front-loaded signals. The label "designature" means that the data of which the VSP signal was extracted had the source signature removed. The underlying model was derived from the Brugdan I well logs. The logs were upscaled to 0.7620-m-thick layers. The vertical lines attached to the VSP wavelet curve show the uncertainties in the phase estimates that were obtained with different analysis window lengths (100-500 ms). At the top, the kurtosis sensitivity measure $S_{D}$ is plotted for the VSP (no designature) curve using a 100-ms-long time window. The source waveforms used in the modeling are given in panel (b) in the same color as the corresponding phase curves in panel (a). The evolved waveforms at $1899 \mathrm{~m}$ depth are displayed in panel (c). 
wavelets with 5 and $10 \mathrm{~Hz}$ low-cut frequencies show phase delays within the topmost $250 \mathrm{~m}$ of the basalts that are very similar to the phase delays observed in the VSP (see Figure 9). At $1430 \mathrm{~m}$ depth, the phase change (delay) of the extracted VSP signal is $90^{\circ} \pm 40^{\circ}$ and the phase delay in the VSP signal is $99^{\circ} \pm 34^{\circ}$. High-phase angle uncertainties co-occur within the 1180-1450 m depth interval known to cause strong wave interference effects on the first arrival. We show error bars for the phase curve that was obtained using the VSP signal, which was extracted from data that include the source signature (see Figures 9a and 12a). The phase uncertainties for the data are of the same order as for the synthetic. The phase curves related to the wavelets with the 5 and $10 \mathrm{~Hz}$ low-cut frequencies also show large apparent phase uncertainties of up to $60^{\circ}$ in the 1180-1450 m depth interval. All other phase curves have smaller phase errors $\left(<5^{\circ}\right)$. Despite the high phase-angle uncertainties, the apparent phase-angle curves show exponential-like changes below the top basalt in Brugdan I.

\section{PREDICTED PHASE SHIFTS BY VERTICAL- SEISMIC-PROFILE-DERIVED EFFECTIVE $Q$}

Intrinsic seismic absorption can be specified via an intrinsic seismic quality factor $Q_{\text {int }}$, which will lead to phase shifts in the signals. We can see this when we replace $k(\omega)=\omega / c(\omega)+i \alpha(\omega)$ and $c(\omega)=|\omega| /\left(2 \alpha(\omega) Q_{\text {int }}\right)$ in equation 4 , where $c$ is the phase velocity. Equation 4 is also the basis for inverse- $Q$ filtering (e.g., Robinson, 1982; Hargreaves and Calvert, 1991). We study such phase shifts by incorporating the intrinsic attenuation via the Kolsky-Futterman near-constant $Q$ model (Wang, 2008). Effective medium velocities of the Brugdan I basalt sequence were used as background velocities along with a $Q_{\text {int }}$. The kurtosis and Fourier approaches were used to estimate the apparent phase of the signals propagating through the medium. The intent of predicting phase shifts with a certain $Q$ value is to assess whether a good inverse (phase-only) $Q$ compensation can be achieved by a $Q_{\text {eff }}$ at larger depths in the Brugdan I basalt sequence.

Mateeva et al. (2002) compare the properties of the intrinsic and scattering attenuation operators in finely layered geologies with synthetic data. They conclude that an effective attenuation operator would underestimate the phase-lag of the first arrivals in a medium with strong reflectivities and free surface, but it would be usable for inverse filtering without a free surface. We believe that our VSP case is more akin to the no-free-surface model of Mateeva et al. (2002). The basalt layers are deep below the free surface, and the sediments above the top basalt are fairly homogeneous (not thinly layered). We used the average, VSP-derived, effective quality factor of $Q_{\text {eff }}=25$ from Schuler et al. (2014) within the 2302 $3163 \mathrm{~m}$ depth interval for our modeling and use $Q_{\text {eff }}$ as $Q_{\text {int }}$. Wavelets from Figure $12 \mathrm{~b}$ were prepared as source signals. We verified that the peak frequency of the analyzed signals was smaller than their bandwidth for the kurtosis approach to work. The predicted phase delays due to a $Q_{\text {eff }}=25$ range from $6^{\circ}$ to $17^{\circ}$ over the 2302-3163 m depth interval with few phase delays observed when the signals contain more low-frequency content. Although every test wavelet led to slightly different phase delays per unit depth, they all showed linear phase changes with depth.

A regression line was fitted through the phase estimates between 2302 and $3163 \mathrm{~m}$ depth in Figure 9a. An overall phase delay of $10^{\circ} \pm 3^{\circ}$ was calculated over the 2302-3163 m depth interval, which fits well into the range of predicted phase delays. We were not able to do the same analysis over the topmost basalt sequence because no frequency-independent $Q_{\text {eff }}$ could be estimated there.

\section{DISCUSSION: PHASE ESTIMATES OF SYNTHETIC SEISMOGRAMS}

The shape of the phase-angle curve could be reproduced well with 1D modeling within the topmost $250 \mathrm{~m}$. The phase changes, calculated from the synthetic seismograms (without absorption) using VSP signals as source waveforms, were similar to the phase shifts that were computed from the VSP data within the top interval. The positive phase shifts between 1600 and 1800 m depth in Figure 9a were not observed in Figure 12a. However, they lie within the phase uncertainty estimates of each curve and thus are not interpreted.

Elastic modeling within the topmost basalt interval also showed that it would be difficult to compensate the phase change with a constant $Q_{\text {eff }}$ value at Brugdan I. Because the phase-angle curves change in an exponential-like manner, they could only be matched with an inverse, time-variant, phase-only $Q$ compensation. The phase varies then in linear segments in each layer of constant $Q$, but it may not vary linearly over such an inhomogeneous medium.

To perform a good phase-only compensation with a combined effective attenuation factor, the intrinsic and scattering attenuation operators need to have similar properties. Mateeva et al. (2002) note that these operator properties are an exponential decay with frequency and minimum phase. They conclude that scattering attenuation is a significantly nonminimum phase in a thin layered medium with high reflectivity and a free surface. Analyzing the frequency spectra of the signals within the topmost basalt sequence in Brugdan I, complex scattering also leads to a nonexponential decay of amplitude with frequency; i.e., the effective quality factor is frequency dependent in the depth interval where complex scattering occurs. The wavenumber $(k)$ times the scale length of the scatterer $(a)$ within the topmost basalt sequence is about $k a=1-1.5$. We estimated the wavenumber $(k)$ from the seismic signals and the scale length of the scatterer $(a)$ from the sonic logs. At a larger depth within the Brugdan I basalt sequence, the impedance contrasts are weaker, the frequency bandwidth of the VSP signals changes only slightly, and $k a<1$. After Aki and Richards (1980), complex scattering is expected mainly when $1<k a<10$, whereas for very large or very small $k a$, one can consider the waves propagating through an effective medium. For our case, we cannot use $k a$ alone to explain when the phase-only compensation works. For example, $k a$ is smaller than one at a larger depth in the Brugdan I basalts, but we are still in a sequence that comprises thin layers that lead to complex wave scattering and apparent attenuation (Schuler et al., 2014). Therefore, we cannot replace such a sequence with an effective medium without absorption.

An alternative explanation for why the phase-only compensation would work only in certain basalt intervals at Brugdan I is the change in the reflectivity strength with depth. Our reasoning here closely follows Mateeva (2003). Considering a time window around the downgoing first arrivals, the signal window contains a mixture of the downgoing wavelet and its reflections from thin layers immediately below the receiver. These reflections are not removed by the up-down wavefield separation and thus contribute to the wavelet reshaping. The contribution by the reflections is superimposed on the transmission filtering effect of the layering between receiver levels. Due to the higher reflectivity strength in the topmost 
basalt interval at Brugdan I, the signal window likely has a stronger contribution from reflections than a signal window for a receiver that is located above an interval with weaker reflectivity strength. Hence, the stronger thin-bed reflections in the topmost basalt interval may have altered the amplitude and phase of the effective apparent attenuation operator in a way that violates its minimumphase property and makes phase-only compensation unfeasible.

\section{CONCLUSIONS}

We have analyzed the evolution of seismic waveforms in two near-offset VSPs starting from sediments above the volcanic sequence to deeper lying basalt flow sequences. The high impedance contrasts between basalt flows and thin interbed sediments cause strong multiple scattering. We focused on measuring the apparent phase changes of the primary arrivals and compared them with the results that were obtained from synthetic seismograms. We could match the main waveform features of the primary downgoing VSP wavefield with synthetic waveforms including the rapid broadening of the signal signature. Thus, non-1D scattering and intrinsic attenuation appear to have a small impact on our waveforms.

A phase-estimation technique was applied to the VSP data that is based on the kurtosis measure. At Brugdan I, we observed rapid apparent phase changes of approximately $100^{\circ}$ within the top $250 \mathrm{~m}$ of the basalt sequence with little change thereafter. More moderate apparent phase changes were observed in the William basalt sequence, where the geology is slightly less stratified and the distribution of high impedance contrasts led to less strong wave interference of the first arrival. Positive and negative phase shifts were measured in both VSPs within the topmost basalt intervals, where complex scattering occurs. Synthetic data were generated to compare the apparent phase estimates with the real data at Brugdan I. The modeled signals showed similar amounts of phase shifts and therefore matched the apparent phase changes of the VSP data well within the error estimates.

Finally, a VSP-derived (frequency-independent) seismic quality factor was used to predict phase shifts at a larger depth in the Brugdan I basalt sequence. Less complex scattering was expected at depth based on the well logs. Here, the predicted phase shifts with depth were similar to the values measured from the VSP. This suggests that the extent to which phase-only compensation in a basalt sequence can be achieved with an effective quality factor $Q_{\text {eff }}$, depends on the signal bandwidth, distribution, strength, and depth of the reflectors.

\section{ACKNOWLEDGMENTS}

We wish to thank Shell UK Ltd. and BP for providing the data sets and for the permission to publish them. The views expressed herein, however, are those of the authors, who are solely responsible for any errors. We thank J. Neep and two anonymous reviewers for critically reading the manuscript. Thanks go to A/S Norske Shell, Schlumberger Gould Research, and the Natural Environment Research Council (grant no. NE/H025006/1) for financial support. St. Edmund's College and the Cambridge Philosophical Society further supported the first author during field work. This is Cambridge University Department of Earth Sciences contribution no. esc.3066.

\section{REFERENCES}

Aki, K., and P. G. Richards, 1980, Quantitative seismology: Theory and methods: W. H. Freeman.

Backus, G. E., 1962, Long-wave elastic anisotropy produced by horizontal layering: Journal of Geophysical Research, 67, 4427-4440, doi: 10.1029/ JZ067i011p04427.

Banik, N. C., I. Lerche, and R. T. Shuey, 1985, Stratigraphic filtering. Part I: Derivation of the O'Doherty-Anstey formula: Geophysics, 50, 27682774, doi: 10.1190/1.1441897.

Bracewell, R. N., 1965, The Fourier transform and its applications: McGraw-Hill Book Co. Inc.

Burridge, R., 1990, Some notes on the effective medium, Technical Report, Schlumberger-Doll Research.

Burridge, R., G. S. Papanicolaou, and B. S. White, 1988, One-dimensional wave propagation in a highly discontinuous medium: Wave Motion, 10, 19-44, doi: 10.1016/0165-2125(88)90004-2.

Claerbout, J. F., 1985, Fundamentals of geophysical data processing: Blackwell Scientific.

de Hoop, M. V., R. Burridge, and H.-W. Change, 1991, Wave propagation with tunneling in a highly discontinuous layered medium: Wave Motion, 13, 307-327, doi: 10.1016/0165-2125(91)90067-X.

Donoho, D., 1981, On minimum entropy deconvolution, in D. Findley, eds., Applied time series analysis II: Academic Press, 565-608.

Edgar, J. A., and J. I. Selvage, 2011, Can thin beds be identified using statistical phase estimation?: First Break, 29, 55-65, doi: 10.3997/1365-2397 .2011009.

Edgar, J. A., and M. van der Baan, 2011, How reliable is statistical wavelet estimation?: Geophysics, 76, no. 4, V59-V68, doi: 10.1190/1.3587220.

Esmersoy, C., 1990, Inversion of P and SV waves from multicomponent offset vertical seismic profiles: Geophysics, 55, 39-50, doi: 10.1190/1.1442770.

Folstad, P. G., and M. Schoenberg, 1992, Low-frequency propagation through fine layering: 62nd Annual International Meeting, SEG, Expanded Abstracts, 1279-1281.

Futterman, W. I., 1962, Dispersive body waves: Journal of Geophysical Research, 67, 5279-5291, doi: 10.1029/JZ067i013p05279.

Hargreaves, N., and A. Calvert, 1991, Inverse $Q$ filtering by Fourier transform: Geophysics, 56, 519-527, doi: 10.1190/1.1443067.

Jerram, D. A., N. Mountney, and H. Stollhofen, 1999, Facies architecture of the Etjo Sandstone Formation and its interaction with the Basal Etendeka Flood Basalts of northwest Namibia: Implications for offshore prospectivity: Geological Society, London, Special Publications, 153, 367-380, doi: 10.1144/GSL.SP.1999.153.01.22.

Kennett, B. L. N., 1974, Reflections, rays, and reverberations: Bulletin of the Seismological Society of America, 64, 1685-1696.

Kennett, B. L. N., 1983, Seismic wave propagation in stratified media: Cambridge University Press.

Lazear, G. D., 1993, Mixed-phase wavelet estimation using fourth-order cumulants: Geophysics, 58, 1042-1051, doi: 10.1190/1.1443480.

Levy, S., and D. W. Oldenburg, 1987, Automatic phase correction of common-midpoint stacked data: Geophysics, 52, 51-59, doi: 10.1190/ 1.1442240 .

Longbottom, J., A. T. Walden, and R. E. White, 1988, Principles and application of maximum kurtosis phase estimation: Geophysical Prospecting, 36, 115-138, doi: 10.1111/j.1365-2478.1988.tb02155.x.

Martini, F., and C. J. Bean, 2002, Interface scattering versus body scattering in subbasalt imaging and application of prestack wave equation datuming: Geophysics, 67, 1593-1601, doi: 10.1190/1.1512750.

Mateeva, A., 2003, Thin horizontal layering as a stratigraphic filter in absorption estimation and seismic deconvolution: Doctoral thesis, Colorado School of Mines.

Mateeva, A., D. Hart, and S. MacKay, 2002, Apparent attenuation from short-period multiples and intrinsic absorption in the seismic wavelet model: 72nd Annual International Meeting, SEG, Expanded Abstracts, 2198-2201.

Newman, P., 1973, Divergence effects in a layered earth: Geophysics, 38 , 481-488, doi: 10.1190/1.1440353.

O'Doherty, R. F., and N. A. Anstey, 1971, Reflections on amplitudes: Geophysical Prospecting, 19, 430-458, doi: 10.1111/j.1365-2478.1971 .tb00610.x.

Peacock, K. L., and S. Treitel, 1969, Predictive deconvolution: Theory and practice: Geophysics, 34, 155-169, doi: 10.1190/1.1440003.

Resnick, J. R., I. Lerche, and R. T. Shuey, 1986, Reflection, transmission, and the generalized primary wave: Geophysical Journal of the Royal Astronomical Society, 87, 349-377, doi: 10.1111/j.1365-246X.1986 .tb06628.x.

Richards, P. G., and W. Menke, 1983, The apparent attenuation of a scattering medium: Bulletin of the Seismological Society of America, 73, 1005-1021.

Robinson, J. C., 1982, Time-variable dispersion processing through the use of "phased" sinc functions: Geophysics, 47, 1106-1110, doi: 10.1190/1 .1441374 
Roenz, B., and H. G. Strohe, 1994, Lexikon Statistik: Gabler Verlage.

Schoenberger, M., and F. K. Levin, 1974, Apparent attenuation due to intrabed multiples: Geophysics, 39, 278-291, doi: 10.1190/1.1440427.

Schoenberger, M., and F. K. Levin, 1978, Apparent attenuation due to intrabed multiples, II: Geophysics, 43, 730-737, doi: 10.1190/1.1440849.

Schuler, J., P. A. F. Christie, and R. S. White, 2014, Borehole study of compressional and shear attenuation of basalt flows penetrated by the Brugdan and William boreholes on the Faroes shelf: Geophysical Prospecting, 62, 315-332, doi: 10.1111/1365-2478.12087.

Shapiro, S. A., and S. Treitel, 1997, Multiple scattering of seismic waves in multilayered structures: Physics of the Earth and Planetary Interiors, 104, 147-159, doi: 10.1016/S0031-9201(97)00043-5.

Simm, R., and R. White, 2002, Tutorial: Phase, polarity and the interpreter's wavelet: First Break, 20, 277-281, doi: 10.1046/j.1365-2397 .2002.00277.x

Spencer, T. W., C. M. Edwards, and J. R. Sonnad, 1977, Seismic wave attenuation in nonresolvable cyclic stratification: Geophysics, 42, 939-949, doi: 10.1190/1.1440773.

Stewart, R. R., P. D. Huddleston, and T. K. Kan, 1984, Seismic versus sonic velocities: A vertical seismic profiling study: Geophysics, 49, 1153-1168, doi: $10.1190 / 1.1441745$
Ulrych, T. 1999, The whiteness hypothesis: Reflectivity, inversion, chaos, and Enders: Geophysics, 64, 1512-1523, doi: 10.1190/1.1444655.

van der Baan, M., 2008, Time-varying wavelet estimation and deconvolution by kurtosis maximization: Geophysics, 73, no. 2, V11-V18, doi: 10.1190/ 1.2831936

Walden, A. T., and J. W. J. Hosken, 1985, An investigation of the spectral properties of primary reflection coefficients: Geophysical Prospecting, 33, 400-435, doi: 10.1111/j.1365-2478.1985.tb00443.x.

Walden, A. T., and J. W. J. Hosken, 1986, The nature of the non-Gaussianity of primary reflection coefficients and its significance for deconvolution: Geophysical Prospecting, 34, 1038-1066, doi: 10.1111/j.1365-2478.1986 .tb00512.X.

Wang, Y., 2008, Seismic inverse $Q$ filtering: Blackwell Publishing.

White, R. E., 1988, Maximum kurtosis phase correction: Geophysical Journal, 95, 371-389, doi: 10.1111/j.1365-246X.1988.tb00475.x.

Widess, M. B., 1973, How thin is a thin bed?: Geophysics, 38, 1176-1180, doi: 10.1190/1.1440403.

Ziolkowski, A., P. Hanssen, R. Gatliff, H. Jakubowicz, A. Dobson, G. Hampson, X.-Y. Li, and E. Liu, 2003, Use of low frequencies for subbasalt imaging: Geophysical Prospecting, 51, 169-182, doi: 10.1046/j .1365-2478.2003.00363.x. 